Journal of the Operations Research Society of Japan

Vol. 30, No. 3, September 1987

\title{
A POLYNOMIAL-TIME DUAL SIMPLEX ALGORITHM FOR THE MINIMUM COST FLOW PROBLEM
}

\author{
Akira Nakayama \\ Otaru University of Commerce
}

(Received October 11, 1985; Final May 11, 1987)

\begin{abstract}
Recently, Ikura and Nemhauser proposed a polynomial-time algorithm for the transportation problem of the Hitchcock type by the dual simplex method. The Ikura-Nemhauser algorithm can also solve the general minimum cost flow problem by reducing the minimum cost flow problem to a Hitchcock problem. In this paper, instead of such a reduction, we show a polynomial-time dual simplex algorithm for finding a minimum cost flow in a two-terminal capacitated network by applying Ikura and Nemhauser's idea directly to general two-terminal networks.
\end{abstract}

\section{Background}

Dantzig [3] devised the simplex method for linear programming problems and showed a way of specializing the simplex method to the minimum cost flow problem, a special case of linear programming problems. Cunningham [2] recently developed a nice anticycling rule for the network simplex method. Later primal dual method for network flow problems has been proposed by Ford and Fulkerson [5] and Iri [10]. Fulkerson [6] also proposed an approach called out-of-kilter method to the minimum cost flow problem. Other algorithms, primal methods, to find a minimum cost flow are seen in the work by Balinski and Gomory [1] and Klein [11]. However, all of these methods are not polynomial-time algorithms. In 1972, Edmonds and Karp [4] gave a polynomial-time algorithm for the first time by introducing a new technique, i.e., capacity-scaling method. Lawler [12] also showed a polynomial-time algorithm by employing the out-of-kilter method with capacity scaling •

Recently, Ikura and Nemhauser [8,9] gave a polynomial-time dual simplex 
algorithm for the Hitchcock transportation problem. Furthermore, Tardos [15] has recently shown a strongly polynomial algorithm for the minimum cost flow problem. Namely, she has affirmatively solved the open problem posed by Edmonds and Karp in 1972. This is the problem of finding an algorithm for the minimum cost flow problem which requires polynomial time in the numbers of vertices and arcs of the underlying graph and independent of the sizes of the cost and capacity functions. Very recently, Orlin [13] and Fujishige [7] have devised faster algorithms than Tardos's.

In this paper, we present a polynomial-time dual simplex algorithm for finding a minimum cost flow in a two-terminal capacitated network by applying Ikura and Nemhauser's idea [8]. Our algorithm is not a strongly polynomial-time one, but it is expected that the proposed dual simplex method is a practically efficient one as is the simplex method for general linear programming problems.

Since the minimum cost flow problem can be reduced to a Hitchcock problem ([5]), it can be solved by the Ikura-Nemhauser algorithm by reducing it to a Hitchcock problem. It would, however, be pointless to do so from the computational point of view. It would be worth presenting a version. of the Ikura-Nemhauser algorithm which can directly be applied to general two-terminal networks. As will be seen later, it is not so straightforward to devise such a version of the Ikura-Nemhauser algorithm and to estimate the computational complexity.

\section{Minimum Cost Flow Problem}

Let $G=\left(V, A, \partial^{+}, \partial^{-}\right)$be a (directed) graph. $V$ and $A$ denote the set of vertices and the set of arcs of $G$, respectively. $\partial^{+}$(resp. $\partial^{-}$) is a function from $A$ to $V$ and for each arc $a, \partial^{+} a$ (resp. $\partial^{-} a$ ) denotes the initial (resp. terminal) vertex of $a$. For simplicity, we also write $G=(V, A)$ in place of $G=\left(V, A, \partial^{+}, \partial^{-}\right)$. For two graphs $G_{i}=\left(V_{i}, A_{i}\right)(i=1,2), G_{1} \cup G_{2}$ is defined by $G_{1} \cup G_{2}=$ $\left(V_{1} \cup V_{2}, A_{1} \cup A_{2}\right)$, and $G_{1} \subset G_{2}$ means that $G_{1}$ is the subgraph of $G_{2}$. For a graph $G=(V, A)$, the set $R$ of real numbers, given nonnegative upper capacity function $b: A \rightarrow R$ and cost function $c: A \rightarrow R$, let $N=(G=(V, A), b, c)$ be a network. Throughout this paper, we will employ the following rule of expressions in algorithms, propositions and every part of this paper. When we write as $X^{ \pm}=Y^{\mp} \pm Z^{ \pm}$, we mean two relations $X^{+}=Y^{-}+Z^{+}, X^{-}=Y^{+}-Z^{-}$. Then the minimum cost flow problem (P) is formulated as follows. 
(P): $\min \Sigma\{c(a) x(a): a \varepsilon A\}$

$$
\begin{array}{ll}
\sum\left\{x(a): a \varepsilon \delta^{+} v\right\}-\sum\left\{x(a): a \varepsilon \delta^{-} v\right\}=0 & (v \varepsilon V), \\
0 \leqq x(a) \leqq b(a) & (a \varepsilon A) .
\end{array}
$$

Here, $\delta^{+}{ }_{v}\left(\right.$ resp. $\delta^{-} v$ ) is defined as $\delta^{+}{ }_{V=}\left\{a \varepsilon A: \partial^{+} a=v\right\}$ (resp. $\delta^{-}{ }_{v}=\{a \varepsilon A$ : $\left.\left.\partial^{-} a=v\right\}\right)$. For a network $N$, a function $x: A \rightarrow R($ or $(x(a): a \varepsilon A)$ ) satisfying $(1.1)$ is called a circulation or a flow in $N$. If a circulation $x$ also satisfies (1.2), then $x$ is called feasible. Without loss of generality, we assume that $b(a)>0$ for all acA and that $G$ is strongly connected.

2. Dual Simplex Method for the Minimum Cost Flow Problem

\subsection{Potential and Circulation}

The vector $(p(v): v \varepsilon V$ ) ( or simply $p$ ) is called a potential, where the value $p(v)$ is given for each vertex $v \varepsilon V$. First, our particular potential $p$ is determined by the next operation, which is called Procedure $\operatorname{POT}\left(T_{r}\right)$, where $T_{r}$ is a spanning tree with root $r$.

Procedure $\operatorname{POT}\left(T_{r}\right)$

Step 1: Let $p(r)=0$.

Step 2: Calculate $p$ such that $c(a)+p\left(\partial^{+} a\right)-p\left(\partial^{-} a\right)=0$ for any $a \varepsilon A\left(T_{r}\right)$.

Procedure $\mathrm{FLO}\left(p, T_{r}\right)$ shown below finds a circulation in the network $N$, where $p$ is the potential obtained from Procedure POT $\left(T_{r}\right)$.

Procedure $\mathrm{FLO}\left(p, T_{r}\right)$

Step 1: (1.i) If $A-A\left(T_{r}\right)=\phi$, then we set $(x(a): a \varepsilon A)=0$ and stop. (1.ii) For each arc $a \varepsilon A-A\left(T_{r}\right)$, if $c(a)+p\left(\partial^{+} a\right)-p\left(\partial^{-} a\right) \geqq 0$, then we put $x(a)=0$. Otherwise, we put $x(a)=b(a)$.

Step 2: Decide $\left(x(a): a \varepsilon A\left(T_{r}\right)\right)$ so that the condition (1.1) may hold by using $\left(x(a): a \varepsilon A-A\left(T_{r}\right)\right)$ in Step 1 .

Examp 1e-1: In Fig.2, the network $N=(G=(V, A), b, c)$ has $V=\{1,2,3,4\}$ and $A=\left\{a_{i}\right.$ : $1 \leqq i \leqq 6\}$. For each arc $a_{i}$, the ordered pair attached to $a_{i}$ is $\left(c\left(a_{i}\right), b\left(a_{i}\right)\right)$. Let $T_{1}$ be a spanning tree in Fig. 3 whose arcs consist of three waving arrows $a_{2}, a_{3}, a_{5}$, where vertex 1 is the root. By Procedures $\operatorname{POT}\left(T_{r}\right)$ and $\operatorname{FLO}\left(p, T_{r}\right)$, we can find a potential $p$ and a circulation $x$ of the network $N$ in the same figure. 


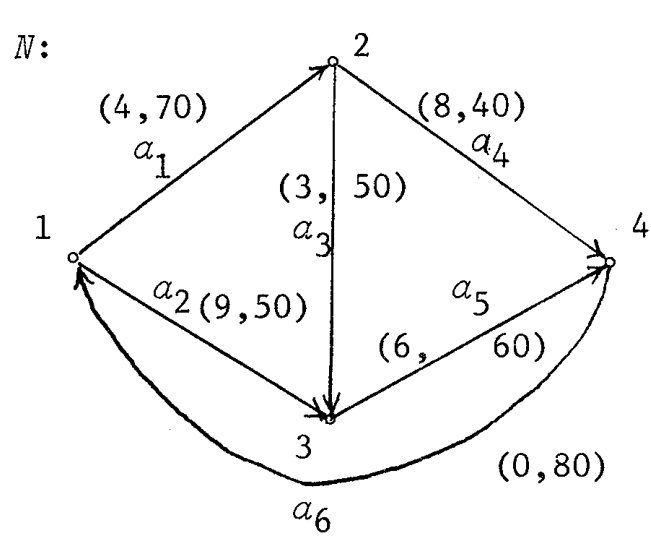

Fig. 2

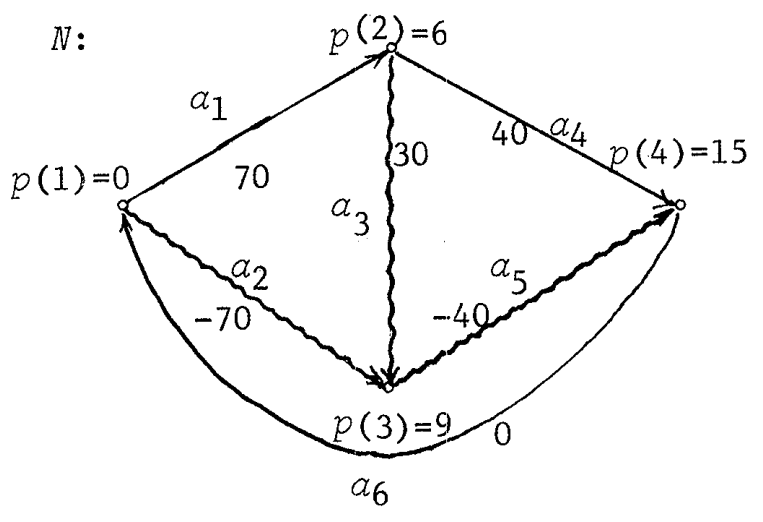

Fig. 3

\subsection{Pivot Operation}

In order to describe the dual simplex method, we define the dual problem (DP) of the primal problem (P) as follows.

$$
\begin{array}{rlrl}
(\mathrm{DP}): & \max -\Sigma\{b(a) \gamma(a): a \varepsilon A\}, & \\
& p\left(\partial^{-} a\right)-p\left(\partial^{+} a\right)+\lambda(a)-\gamma(a)=c(a) & & (a \varepsilon A), \\
& \lambda(a) \geqq 0, \gamma(a) \geqq 0 & & (a \varepsilon A), \\
& p(v) \text { is a free variable } & & (v \varepsilon V) .
\end{array}
$$

Then we have the following complementary slackness condition (CS) for the primal (P) and the dual (DP):

(CS): For any aعA,

$$
\begin{aligned}
& \lambda(a)=0 \text { if } x(a)>0, \\
& x(a)=0 \text { if } \lambda(a)>0, \\
& x(a)=b(a) \text { if } \gamma(a)>0 .
\end{aligned}
$$

The next proposition is well known.

Proposition 2.1. If the feasible solution $x(a)(a \varepsilon A)$ of the primal problem (P) and the feasible one $\lambda(a), \gamma(a)(a \varepsilon A), p(v)(v \varepsilon V)$ of the dual (DP) satisfy condition (CS), then $x(a)$ (aEA) is an optimal solution of (P) and $\lambda(a), \gamma(a)(a \varepsilon A), p(v)(v \varepsilon V)$ is an optimal solution of (DP).

Moreover the reverse is also true. $\square$

Note that this fact still holds even if condition (CS) is replaced with the next one:

$$
\begin{aligned}
(\mathrm{CS})^{*}: \text { For any } a \varepsilon A, \\
\qquad p\left(\partial^{-} a\right)-p\left(\partial^{+} a\right) \leqq c(a) \text { if } x(a)=0,
\end{aligned}
$$




$$
\begin{aligned}
& p\left(\partial^{-} a\right)-p\left(\partial^{+} a\right)=c(a) \text { if } 0<x(a)<b(a), \\
& p\left(\partial^{-} a\right)-p\left(\partial^{+} a\right) \geqq c(a) \text { if } x(a)=b(a) .
\end{aligned}
$$

Now we show how the pivot operations of the dual simplex method are realized on the network $N$. A leaf branch $B_{a}$ with respect to $T_{r}$ and $a \varepsilon A\left(T_{r}\right)$ is defined as the connected component of $T_{r}$-a not containing root $r$. Arcs $a \varepsilon A-A\left(T_{r}\right), a^{\prime} \varepsilon A\left(T_{r}\right)$ are called cotree arc, tree arc, respectively. The two sets $A^{+}\left(T_{r}\right)$ and $A^{-}\left(T_{r}\right)$ are defined as

$$
A^{ \pm}\left(T_{r}\right)=\left\{a \varepsilon A\left(T_{r}\right): \partial \pm a \varepsilon V\left(P_{r \partial \mp_{a}}\right)\right\}
$$

where $P_{r \partial^{+} a}$ (resp. $P_{r \partial^{-}}$) is the path joining root $r$ and vertex $\partial^{+} a$ (resp. $\left.\partial^{-} a\right)$ on tree $T_{r}$. Define $E^{+}$and $E^{-}$by

$$
E^{ \pm}=\left\{a \varepsilon A^{ \pm}\left(T_{r}\right): x(a)>b(a)\right\} \cup\left\{a \varepsilon A^{\mp}\left(T_{r}\right): x(a)<0\right\} .
$$

For a number $\rho$ and an arc $a \varepsilon E^{ \pm}$, Operation $1\left(p, T_{r}, \rho, a\right)$ given below determines a new potential denoted by $p$ again.

Operation $1\left(p, T_{r}, \rho, a\right)$ :

For any vertex $v \varepsilon V\left(B_{a}\right)$, set $p(v)=p(v)+\rho$.

By the way, in order to complete the dual simplex method, this value must be determined so that the new potential $p$ and the circulation $x$ given by Procedure $\mathrm{FLO}\left(p, T_{r}\right)$ may satisfy the condition $(\mathrm{CS})^{*}$. Here, for an arc eeA and a potential $p$, let $\tau(p, e)$ be the reduced cost given by $\tau(p, e)=c(e)+$

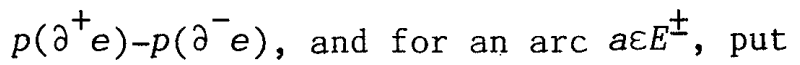

$$
\begin{aligned}
& S_{a}^{ \pm}=\left\{e \varepsilon H_{a}^{ \pm}: \tau(p, e) \geqq 0, x(e)=0\right\}, \\
& T_{a}^{\mp}=\left\{e \varepsilon H_{a}^{\mp}: \tau(p, e) \leqq 0, x(e)=b(e)\right\}
\end{aligned}
$$

where $H_{a}^{+}$and $H_{a}^{-}$are defined as

$$
H_{a}^{ \pm}=\left\{e \varepsilon A-A\left(T_{r}\right): \partial^{ \pm} e \varepsilon V\left(T_{r}\right)-V\left(B_{a}\right), \partial^{F_{e}} \in V\left(B_{a}\right)\right\} .
$$

Then Operation $2\left(a, S_{a}^{ \pm}, T_{a}^{\mp}\right)$ given below decides the value of $\rho$.

Operation $2\left(a, S_{a}^{ \pm}, T_{a}^{\mp}\right)$ :

Find the value $\rho$ by

$\rho= \pm \min \left\{\min \left\{\tau(p, e): e \varepsilon S_{a}^{ \pm}\right\}, \min \left\{-\tau(p, e): e \varepsilon T_{a}^{\mp}\right\}\right\}$

and let $a_{\rho}$ be the arc which attains the minimum value $\rho$.

By each pivot operation, a tree arc breaking the capacity constraint (1.2) is to be replaced by some cotree arc so that the condition (CS)* is preserved after each pivot operation. When the potential $p$ and the circulation $x$ obtained in Section 2.1 are given for the network $N$, the dual simplex method is stated as follows.

Dual simplex method

Step 1: If $E^{+} \cup E^{-}=\phi$, then $x$ is a minimum cost flow, and the algorithm 
terminates.

Step 2: Choose an arc $a \varepsilon E^{\ddagger}$. Then find the value $\rho$ and an arc $a_{\rho}$ by Operation $2\left(a, S_{a}^{ \pm}, T_{a}^{\mp}\right)$.

Step 3: (3.i) Renew the potential by Operation $1\left(p, T_{r}, \rho, a\right)$.

(3.ii) Set $T_{r}=T_{r}-a+a \rho$. Then renew the circulation by Procedure FLO $\left(p, T_{r}\right)$, and return to Step 1 .

\section{Refinement of Dual Simplex Method}

In Section 3.1, we introduce the procedure called Tree Partitioning which decides the tree arc taken in the pivot operation. This is a key operation for showing the polynomial running time. Section 3.2 gives a deficiency function used for proving polynomiality of our algorithm described in Section 3.3.2. Trimming operation in Section 3.3.1 is implemented as a preparation before pivot operations.

\subsection{Tree Partitioning}

We divide the rooted spanning tree $T_{r}$ into a collection of disjoint subtrees, and partition them into three classes. This procedure, called Tree Partitioning, is implemented by Procedure TP $\left(T_{r},\left(x(a): a \varepsilon A\left(T_{r}\right)\right)\right.$ ) described below. In the dual simplex method stated in Section 2, pivot operations may be repeated infinitely. However, Tree Partitioning decides the right arc taken in the pivot operation so that the algorithm may run in polynomial time as we will see later. For the circulation $x$ obtained in Section 2.1, we assume that the number $z_{r}$ defined by

$$
z_{r}=\sum\left\{x(a): a \varepsilon \delta^{+} r\right\}-\Sigma\left\{x(a): a \varepsilon \delta^{-} r\right\}
$$

is assigned to the root $r$. We have $z_{r}=0$ at the present time. Then Procedure $\mathrm{TP}\left(T_{r},\left(x(a): a \varepsilon A\left(T_{r}\right)\right)\right)$ is given as follows.

Procedure TP $\left(T_{r},\left(x(a): a \varepsilon A\left(T_{r}\right)\right)\right)$

(P0): Set $\underline{\mathscr{D}}^{+}=\phi, \underline{\mathscr{D}}^{-}=\phi$ and $\underline{\mathcal{N}}=\phi$.

(P1): If $0 \leqq x(a) \leqq b(a)$ for each $a \varepsilon A\left(T_{r}\right)$, then put

$$
\begin{array}{ll}
\underline{\mathcal{N}}=\underline{\mathcal{N}} \cup\left\{T_{r}\right\} & \text { if } z_{r}=0, \\
\underline{\mathscr{D}}^{-}=\mathscr{D}^{-} \cup\left\{T_{r}\right\} & \text { if } z_{r}>0, \\
\underline{D}^{+}=\underline{D}^{+} \cup\left\{T_{r}\right\} & \text { if } z_{r}<0, \\
\tilde{d}\left(T_{r}\right)=\left|z_{r}\right| &
\end{array}
$$

and stop.

(P2): Choose an arc $a^{*} \varepsilon E^{\ddagger}$ and a leaf branch $B_{a^{*}}$ of $T_{r}$ such that $0 \leqq x(a) \leqq b(a)$ for any $a \varepsilon A\left(B_{a^{*}}\right)$. Then set

$$
\underline{D}^{\mp}=\underline{D}^{\mp} \cup\left\{B_{a} *\right\},
$$


$h= \begin{cases}-x\left(a^{*}\right) & \text { if } a^{*} \varepsilon\left(E^{+} \cap A^{-}\left(T_{r}\right)\right) \cup\left(E^{-} \cap A^{+}\left(T_{r}\right)\right), \\ x\left(a^{*}\right)-b\left(a^{*}\right) & \text { if } a^{*} \varepsilon\left(E^{+} \cap A^{+}\left(T_{r}\right)\right) \cup\left(E^{-} \cap A^{-}\left(T_{r}\right)\right) .\end{cases}$

(P3): If $\underline{\mathcal{D}}^{+} \cup \underline{\mathscr{D}}=\phi$, then we end this procedure. Otherwise, choose $Q \varepsilon \underline{\mathcal{D}}$, and set

$$
\begin{aligned}
& F^{ \pm}=\left\{a \varepsilon A^{ \pm}\left(T_{r}\right): x(a)<b(a)\right\} \cup\left\{a \varepsilon A^{\mp}\left(T_{r}\right): x(a)>0\right\}, \\
& Z^{ \pm}=\left\{a \varepsilon A^{ \pm}\left(T_{r}\right): x(a)=b(a)\right\} \cup\left\{a \varepsilon A^{\mp}\left(T_{r}\right): x(a)=0\right\} \text {. } \\
& \text { (P3.i) If } A(Q) \cap Z^{ \pm}=\phi \text {, then put } \tilde{d}(Q)=h \text {, and go to (P4). } \\
& \text { Otherwise, choose a leaf branch } B_{a} \text { ". of } Q \text { such that } \\
& A\left(B_{a}{ }^{\prime \prime}\right) \subset F^{ \pm} \text {for } a^{\prime \prime} \varepsilon A(Q) \cap Z^{ \pm} \text {. Then we set } \\
& \underline{\mathcal{N}}=\underline{\mathcal{N}} \cup\left\{B_{a} \|\right\}, \\
& \underline{D}^{ \pm}=\underline{D}^{ \pm}-\{Q\} \text {, } \\
& Q=Q-V\left(B_{a}\right) \text {, } \\
& \underline{D}^{ \pm}=\underline{D}^{ \pm} \cup\{Q\} \\
& \text { and return to (P3.i). }
\end{aligned}
$$

(P4): For the arc $a^{*} \varepsilon E^{ \pm}$obtained in (P2), carry out $\operatorname{ADJUST}\left(T_{r}, a^{*},(x(a)\right.$ : $\left.\left.\operatorname{a\varepsilon A}\left(T_{r}\right)\right), z_{r}\right)$ and return to $(\mathrm{P} 1)$.

Here, the meaning of the function $\tilde{d}$ appearing in (P1) and (P3) will be made clear in Section 3.2, while $\operatorname{ADJUST}\left(T_{r}, a^{*},\left(x(a): a \varepsilon A\left(T_{r}\right)\right), z_{r}\right.$ ) (or simply ADJUST-operation ) in (P4) is as follows.

ADJUST $\left(T_{r}, a^{*},\left(x(a): a \varepsilon A\left(T_{r}\right)\right), z_{r}\right)$

(1.i): For $a^{*} \varepsilon E^{ \pm}$define $\nu$ by

$$
\nu= \begin{cases}-x\left(a^{*}\right) & \text { if } a^{*} \varepsilon A^{\mp}\left(T_{r}\right), \\ -b\left(a^{*}\right)+x\left(a^{*}\right) & \text { if } a^{*} \varepsilon A^{\ddagger}\left(T_{r}\right) .\end{cases}
$$

(1.ii): Let $T_{r}=T_{r}-V\left(B_{a^{*}}\right)$ and $P_{r u}$ be the path of the new tree $T_{r}$ such that $u \varepsilon\left\{\partial^{+} a^{*}, \partial^{-} a^{*}\right\}$. Then adjust $\left(x(a): a \varepsilon A\left(T_{r}\right)\right)$ and $z_{r}$ in the following way •

$$
\begin{array}{ll}
x(a)=x(a)-\nu & \text { if } a \varepsilon A^{ \pm}\left(T_{r}\right) \cap A\left(P_{r u}\right), \\
x(a)=x(a)+\nu & \text { if } a \varepsilon A^{\mp}\left(T_{r}\right) \cap A\left(P_{r u}\right), \\
z_{r}=z_{r} \mp \nu . &
\end{array}
$$

After carrying out Procedure $\operatorname{TP}\left(T_{r},\left(x(a): a \varepsilon A\left(T_{r}\right)\right)\right)$, for $\subseteq \varepsilon\left\{\underline{D}^{+}, \underline{D}, \underline{\mathcal{N}}\right\}$ we construct the new class $C$ by doing the following JOIN $\subseteq$ ) ( or simply JOIN-operation ) which combines the members of $\underline{C}$.

$\operatorname{JOIN}(\underline{c}$ )

Step 1: If $|\underline{C}| \leqq 1$, then $\operatorname{set} C=\underline{C}$ and stop.

Step 2: If there exist an $\operatorname{arc} a \varepsilon A\left(T_{r}\right)$ and two distinct members $Q, Q^{\prime}$ of 
C connected by the arc $a$, then set

$$
\begin{aligned}
& \underline{c}=\underline{c}-\left\{Q, Q^{\prime}\right\}, \\
& \underline{c}=\underline{c} \cup\left\{Q \cup Q^{\prime}+a\right\}
\end{aligned}
$$

and return to Step 1. Otherwise, put $\mathcal{C}=\underline{C}$ and stop.

An example of these operations will be shown in Example-2 of the next section.

\subsection{Deficiency Functions}

When we implement Tree Partitioning, three classes $\underline{D}^{+}, \underline{D}$ and $\underline{\mathcal{N}}$ are obtained in Section 3.1. Moreover, for the set $R_{+}$of nonnegative numbers, a function $\tilde{d}: \underline{D}^{+} \cup \underline{\mathscr{D}} \rightarrow R_{+}$is introduced in Tree Partitioning. This function $\tilde{d}$ is called a deficiency function, which can be regarded as the measure of the primal infeasibility. We define a function $d$ for the members of $D=D^{+} \cup D^{-}$, also called a deficiency function, by

$$
d\left(Q^{*}\right)=\sum\left\{\tilde{d}(Q): Q \varepsilon \underline{D}^{ \pm}, Q \subset Q^{*}\right\}
$$

$$
\left(Q^{*} \varepsilon \mathscr{g}^{ \pm}\right) \text {. }
$$

The quantity $d(D)$ defined by

$$
d(D)=\sum\left\{d\left(Q^{*}\right): Q^{*} \varepsilon \bigotimes\right\}
$$

is called the deficiency of the circulation $x$ in network $N$.

Example-2: Fig.4 is the same as Fig.2, where the set of solid arcs constitutes a rooted spanning tree $T_{1}$, and the dotted arcs mean cotree arcs. The ordered pair attached to each tree arc a shows $(x(a), b(a))$. We perform Tree Partitioning by using the given $T_{1}$ and $\left(x(a): a \varepsilon A\left(T_{1}\right)\right)$ in Fig.3. In Procedure TP $\left(T_{1},\left(x(a): a \varepsilon A\left(T_{1}\right)\right)\right),(P 1)$ is skipped first. Going to (P2), we have $G \varepsilon \underline{D}^{+}$for graph $G=(\{4\}, \phi)$ because $x\left(a_{5}\right)=-40<0$. For this graph $G$, we have $\tilde{d}(G)=40$ in (P3). Then proceeding to (P4), the values $x(a)\left(a \varepsilon A\left(T_{1}^{\prime}\right)\right)$ will be adjusted by ADJUST-operation, where $T_{1}^{\prime}=T_{1}-\{4\}$. That is, we add to $x$ a flow of 40 from root 1 to arc $a_{5}$ so that we have the subtree $T_{1}^{\prime}$ with the

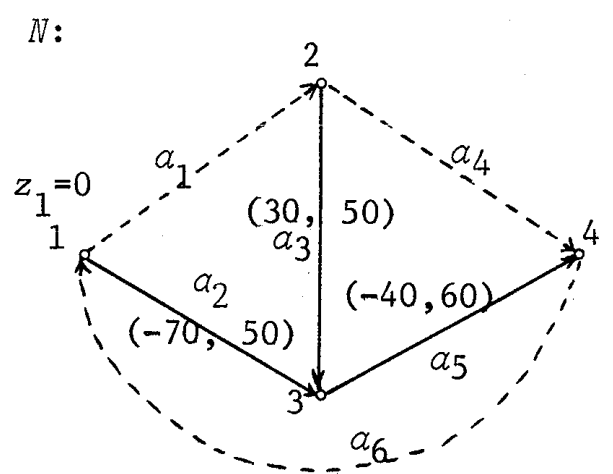

Fig.4

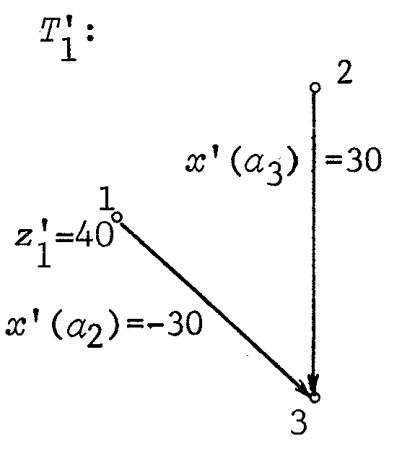

Fig. 5
$T_{1}^{\prime \prime}:$

1

$z_{1}^{\prime \prime}=70$

Fig. 6 
newly values $x^{\prime}(a)\left(\operatorname{a\varepsilon A}\left(T_{1}^{\prime}\right)\right)$ and $z_{1}^{\prime}$ in Fig.5. We apply the above process to $T_{1}^{\prime}$ again. Since $x^{\prime}\left(a_{2}\right)=-30$, we have $G^{\prime}=\left(\{2,3\},\left\{a_{3}\right\}\right) \varepsilon \underline{D}^{+}$and $\tilde{d}\left(G^{\prime}\right)=30$. Let $z_{1}^{\prime \prime}$ be the value obtained by ADJUST $\left(T_{1}^{\prime}, a_{2},\left(x^{\prime}(a): a \varepsilon A\left(T_{1}^{\prime}\right)\right), z_{1}^{\prime}\right)$, then we have $z_{1}^{\prime \prime}=70$ as in Fig.6. When (P1) is repeated for $T_{1}^{\prime \prime}=T_{1}^{\prime}-\{2,3\}$, we have $T_{1}^{\prime \prime} \varepsilon \mathscr{D}^{-}$and $\tilde{d}\left(T_{1}^{\prime \prime}\right)=70$. These are summarized as follows: $\underline{D}^{+}=\left\{G, G^{+}\right\}, \underline{D}^{-}=\left\{T_{1}^{\prime \prime}\right\}, \underline{\mathcal{N}}=\phi$.

By JOIN-operations, we have $D^{+}=\left\{G \cup G^{\prime}+a_{5}\right\}, D^{-}=\left\{T_{1}^{\prime \prime}\right\}, \mathcal{N}=\phi$.

The deficiencies are given by

$$
\begin{aligned}
& \tilde{d}(G)=40, \tilde{d}\left(G^{\prime}\right)=30, \tilde{d}\left(T_{1}^{\prime \prime}\right)=70, \\
& d\left(G \cup G^{\prime}+a_{5}\right)=\tilde{d}(G)+\tilde{d}\left(G^{\prime}\right)=70, \quad d\left(T_{1}^{\prime \prime}\right)=\tilde{d}\left(T_{1}^{\prime \prime}\right)=70 .
\end{aligned}
$$

Hence, the deficiency $d(D)$ of the circulation $x$ in network $N$ is 140 . Note that the deficiency $d(D)$ is determined by the rooted spanning tree $T_{1}$.

\subsection{Description of Algorithm}

We introduce the following Trimming operation as in [8] which improves the efficiency of the dual simplex method.

\subsubsection{Trimming Operation}

As will be seen later, we choose an arc $a^{*}$, as a pivot operation, such that $B_{a}^{*}=Q$ for some $Q \varepsilon D$. Such an arc $a^{*}$ is called a good arc. In this section, we show that some rooted tree with good arcs can be constructed, if necessary, by Trimming operation given below.

Let $T_{r}$ be a rooted tree with no good arcs, then we can find $Q \varepsilon \mathcal{D}$ such that $Q_{i} \in \mathcal{N}(2 \leqq i \leqq k)$ where $Q_{i}(1 \leqq i \leqq k)$ are components of $T_{r}-V(Q)$ satisfying $\operatorname{r\varepsilon V}\left(Q_{1}\right)$. Such a $Q$ is called an $\mathcal{N}$-surrounded graph while each $Q_{i}(2 \leqq i)$ a surrounding $\mathcal{N}$-graph of $Q$. If we can remove all the surrounding $\mathcal{N}$-graphs by pivot operations, then we have good arcs. Consider a pivot operation for arc $a^{i}$ such that $Q_{i}(i \geqq 2)$ equals a leaf branch $B_{a^{i}}$. Let $a^{*}$ be the tree arc of $T_{r}$ connecting $Q_{1}$ to $Q$ and $\tilde{T}_{r}$ the new rooted spanning tree obtained after the pivot operation. Now, we check whether $a^{*}$ is the good arc of $\tilde{T}_{r}$ or not. If $a^{*}$ is a good arc, then we stop here. Otherwise we search for the $\mathcal{N}$ surrounded graph $\tilde{Q}$ of $\tilde{T}_{r}$ incident to $a^{*}$ and a surrounding $\mathcal{N}$-graph $\tilde{Q}_{i}$ of $\tilde{Q}$. Then we continue the pivot operation for the arc $\tilde{a}^{i}$ such that $\tilde{Q}_{i}$ equals some leaf branch $\tilde{B}_{\tilde{a}^{i}}$ of $\tilde{T}_{r}$. That is, we repeat this process until the arc $a^{*}$ becomes the good arc of the current rooted tree. This procedure is called Trimming operation, implemented so that we may have good arcs. Here, we have the next two problems to be considered.

(3.3) Is the number of pivot operations in Trimming operation finite?

(3.4) Can the pivot operations themselves be carried out?

First, we consider (3.4). Let $Q \varepsilon D$ be an $\mathcal{N}$-surrounded graph and $B_{a}$ ' 
the leaf branch of $T_{r}$ which equals a surrounding $\mathcal{N}$-graph of $Q$. Then we have the next proposition, where $p, x$ are the potential, the circulation obtained in Section 2.1, respectively.

Proposition 3.1. If $Q \varepsilon D^{ \pm}$, then we have an $\operatorname{arc} a_{\rho}$ and

$$
x\left(a_{\rho}\right)= \begin{cases}b\left(a_{\rho}\right) & \left(\partial^{ \pm} a_{\rho} \varepsilon V\left(B_{a^{\prime}}\right)\right), \\ 0 & \left(\partial F_{a_{\rho}} \varepsilon V\left(B_{a^{\prime}}\right)\right) .\end{cases}
$$

Proof: We only prove the case when $Q \varepsilon D^{+}$.

Case 1: $a^{\prime} \varepsilon A^{+}\left(T_{r}\right)$; Since $B_{a} \in \mathcal{N}$ and $Q \varepsilon D^{+}$, we have $x\left(a^{\prime}\right)=b\left(a^{\prime}\right)$ and $c\left(a^{\prime}\right)+p\left(\partial^{+} a^{\prime}\right)-p\left(\partial^{-} a^{\prime}\right)=0 . p\left(\partial^{-} a^{\prime}\right)$ must be increased in order to satisfy $\tilde{x}\left(a^{\prime}\right)=b\left(a^{\prime}\right)$ for a new circulation $\tilde{x}$. This means that we may increase the potential of each vertex of $B_{a^{\prime}}$. Hence, Operation $2\left(a^{\prime}, S_{a^{\prime}}^{+}, T_{a^{\prime}}^{-}\right)$is performed. If $S_{a}^{+} \cup T_{a}^{-}=\phi$, then we have

$$
\begin{array}{ll}
x(a)=b(a) & \left(a \varepsilon H_{a}^{+}\right), \\
x(a)=0 & \left(a \varepsilon H_{a}^{-}\right) .
\end{array}
$$

On the other hand, since $x$ is a circulation, we have

$$
\sum\left\{x(a): a \varepsilon H_{a}^{+}\right\}+x\left(a^{\prime}\right)=\sum\left\{x(a): a \varepsilon H_{a^{\prime}}^{-}\right\} \text {. }
$$

Rearranging the equation (3.7) by substituting (3.5) and (3.6), it follows that $b(a)=0$ for any $a \varepsilon H_{a}^{+}, \cup\left\{a^{\prime}\right\}$, but this is impossible.

Case 2: $a^{\prime} \varepsilon A^{-}\left(T_{r}\right)$; we have $H_{a}^{+}=\phi$ as in Case 1. Though $H_{a^{\prime}}^{-} \cup\left\{a^{\prime}\right\}$ is a directed cut, this contradicts the assumption that the underlying graph of the network $N$ is strongly connected.

\subsubsection{Algorithm}

We present the algorithm for the problem $(P)$, which is the dual simplex method accompanied by Tree Partitionings and Trimming operations.

Algorithm

Step 0: (Initialization)

(0.i): Find a spanning tree $T_{r}$ with root $r$ of the network $N$.

(0.ii): Determine the potential $p$ by Procedure POT $\left(T_{r}\right)$, and calculate the circulation $x$ by Procedure $\mathrm{FLO}\left(p, T_{r}\right)$.

Step 1: (Implementation of Tree Partitioning)

(1.i): Find $\underline{D}^{+}, \underline{\mathscr{D}}$ and $\underline{\mathcal{V}}$ by Procedure $\operatorname{TP}\left(T_{r},\left(x(a): a \varepsilon A\left(T_{r}\right)\right)\right)$.

(1.ii): Find $D^{+}, D^{-}$and $\mathcal{N}$ by the corresponding JOIN-operations.

Step 2: (Preparation for Pivot Operations)

(2.i): If $D=D^{+} \cup D^{-}$is empty, then the algorithm terminates and $x$ is an optimal solution.

(2.ii): If there exist no leaf branches $Q$ of $T_{r}$ such that $Q \varepsilon D$, then go to Step 5 . 
Step 3: (Pivot Operation)

(3.i): Find a leaf branch $B_{a} * \varepsilon D$ of $T_{r}$.

(3.ii): For $B_{a} * \varepsilon D^{ \pm}$, find $a_{\rho}$ and $\rho$ by Operation 2 ( $a^{*}, S_{a^{*}}^{F_{*}}, T_{a_{*}^{*}}^{ \pm_{*}}$.

(3.iii): Renew potential $p$ by Operation 1 ( $\left(p, T_{r}, \rho, a^{*}\right)$. Set $T_{r}=T_{r}-a^{*}+a_{\rho}$ and renew circulation $x$ by Procedure FLO $\left(p, T_{r}^{\prime}\right)$.

Step 4: (Renewal of Tree Partitioning)

Find $\mathscr{D}^{+}, D^{-}$and $\mathcal{N}$ for the new tree $T_{r}$ in the same way as in

Step 1. Then go to Step 2.

Step 5: (Trimming Operation)

(5.i): Choose an $\mathcal{N}$-surrounded graph $Q \varepsilon D$ and its surrounding $\mathcal{N}$-graph $Q_{i}$ such that leaf branch $B_{a} "=Q_{i}$ for some $a^{\prime \prime}$.

(5.ii): Find the arc $a^{*} \varepsilon A\left(T_{r}\right)$ which connects $Q$ to the component of $T_{r}-V(Q)$ containing root $r$.

(5.iii): For $Q \varepsilon D^{ \pm}$, find $a_{\rho}$ and $\rho$ by Operation 2 (a", $S_{a}^{ \pm}, T_{a}^{F^{\prime \prime}}$ ). Renew potential $p$ by replacing $a^{*}$ with $a^{\prime \prime}$ in (3.iii). (The circulation $x$ is unchanged.)

(5.iv): We repeat Tree Partitioning for the new tree $T_{r}=T_{r}-a^{\prime \prime}+a_{\rho}$ and decide $D^{+}, D^{-}$and $\mathcal{N}$ as in Step 1.

$(5 . v)$ : If $\operatorname{arc} a^{*}$ is good, then go to (3.ii). Otherwise, for the new tree $T_{r}$ find both the $\mathcal{N}$-surrounded graph $Q$ incident to $a^{*}$ and its surrounding $\mathcal{N}$-graph $Q_{i}$ such that leaf branch $B_{a}{ }^{\prime \prime}=Q_{i}$ for some $a^{\prime \prime}$. Then go to (5.iii).

4. Estimation of the Computational Complexity of the Algorithm

4.1. Change of the Deficiency and the Classes of Tree Partitioning After a pivot operation, if we repeat Tree Partitioning for the new tree $\tilde{T}_{r}$, then the classes and the deficiency $d(\bigotimes)$ will be different from those of $T_{r}$ in general. Our aim in this section is to show that $d(\bigotimes)$ does not increase in course of the algorithm and to examine the change of the classes by pivot operations. We achieve this aim in the following two subsections $(4.1 .1)$ and $(4.1 .2)$ separately.

\subsubsection{Pivot Operations in Trimming Operations}

It is easy to see that $d(D)$ is unchanged by Trimming operations. Hence, the problem to be considered here is to estimate the number of consecutive Trimming operations. Let $\underline{\tilde{D}}^{+}, \underline{\tilde{D}}$ and $\tilde{\mathcal{X}}$ be the classes of Tree Partitioning for $\tilde{T}_{r}$, and $\tilde{D}^{+}, \tilde{D}^{-}$and $\tilde{\mathcal{N}}$ those obtained by the corresponding JOIN-operations. For the two arcs $a^{\prime \prime}$ and $a_{\rho}$ chosen in (5.i) and (5.iii) of Step 5, define vertices $u, y \varepsilon V-V\left(B_{a} "\right)$ and $v, w \varepsilon V\left(B_{a} "\right)$ by $\{u, v\}=\left\{\partial^{+} a^{\prime \prime}, \partial^{-} a^{\prime \prime}\right\}$, 
$\{w, y\}=\left\{\partial^{+} a_{\rho}, \partial^{-} a_{\rho}\right\}$. (See Fig.7.) Here, assume that $Q \varepsilon D$ is an $\mathcal{V}$-surrounded graph and $B_{a}$ is its surrounding $\mathcal{N}$-graph. The possibility of consecutive Trimming operations consists of the next two cases:

Case $C 1$ : $y \varepsilon V\left(B_{a^{\prime}}\right)$ for some surrounding $\mathcal{N}$-graph $B_{a^{\prime}}$ of $Q$. ( $B_{a^{\prime \prime}}$ is attached to $B_{a^{\prime}}$ by arc $a_{\rho}$ in $\tilde{T}_{r}$ )

Case $C 2$ : yєV $(Q)$. ( $B_{a}$ " is attached to $Q$ by arc $a_{\rho}$ in $\tilde{T}_{r}$.)

In other cases, any surrounding $\mathcal{N}$-graph is to be removed from $Q$ in $\tilde{T}_{r}$. (See Fig.8.) The following proposition, which is easily proved, shows the result for Case $\mathrm{Cl}$.
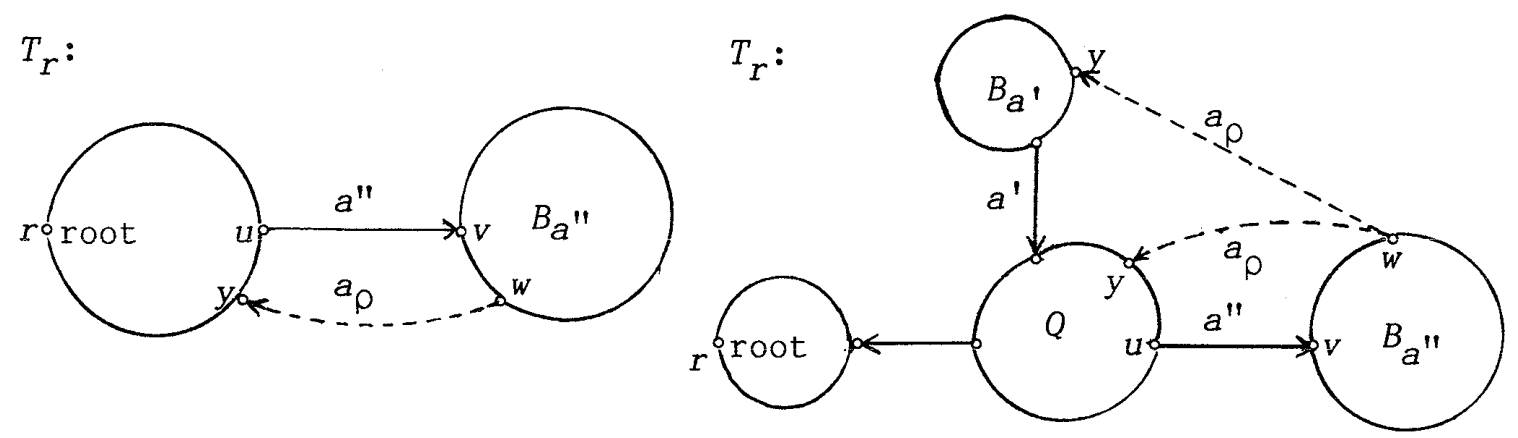

Fig.7

Fig. 8

Proposition 4.1. In Case $\mathrm{Cl}$, we have

$H_{0} \varepsilon \tilde{\mathcal{N}}, \mathcal{D}=\tilde{\mathscr{D}}$ and $\mathcal{N}-\left\{B_{a^{\prime \prime}}, B_{a^{\prime}}\right\}=\tilde{\mathcal{N}}-\left\{H_{0}\right\}$, where $H_{0}=B_{a} " \cup B_{a^{\prime}+a_{\rho}}$.

Before proving Case $\mathrm{C} 2$, first note the following fact:

$\left\{Q^{\prime} \varepsilon \underline{N}: V\left(Q^{\prime}\right) \cap V\left(P P_{V W}\right)=\phi\right\}=\left\{\tilde{Q} \varepsilon \tilde{\mathcal{N}}: V(\tilde{Q}) \cap V\left(\tilde{P}_{V W}\right)=\phi\right\}$,

where $P_{v w}\left(\tilde{P}_{v w}\right)$ is the path of $T_{r}\left(\tilde{T}_{r}\right)$, respectively.

Let $T_{r}^{\prime}=T_{r}-U\left\{V\left(Q^{\prime}\right): Q^{\prime} \varepsilon \underline{\mathcal{N}}, V\left(Q^{\prime}\right) \cap V\left(P_{V W}\right)=\phi\right\}$ and consider $T_{r}^{\prime}$ in place of $T_{r}$ for Case C2. Let $\tilde{T}_{r}^{\prime}$ be the new rooted tree given by the pivot operation of $T_{r}^{\prime}$, and $D^{+}, D^{-}, \mathcal{N}\left(\tilde{D}^{+}, \tilde{D}^{-}, \tilde{\mathcal{N}}\right)$ the partition classes obtained from $T_{r}^{\prime}$ ( $\left.\tilde{T}_{r}^{\prime}\right)$, respectively. Then for an $\mathcal{N}$-surrounded graph $Q$ of $T_{r}^{\prime}$ and its surrounding $\mathcal{N}$-graph which equals leaf branch $B_{a}^{\prime}$ " of $T_{r}^{\prime}$ for some $a^{\prime \prime}$, we have the following proposition.

Proposition 4.2. For $Q \varepsilon D^{ \pm}$in Case C2, we have either (1) or (2).

(1) $H_{1} \varepsilon \tilde{D}^{ \pm}, \quad \mathscr{D}^{ \pm}-\{Q\}=\tilde{D}^{ \pm}-\left\{H_{1}\right\}$ and $\mathcal{N}-\left\{B_{a}^{\prime \prime \prime}\right\}=\tilde{\mathcal{N}}$,

(2) For some leaf branch $\tilde{B}_{e}^{\prime} \varsubsetneqq B_{a}^{\prime}$ " of $\tilde{T}_{r}^{\prime}$,

$$
H_{2} \varepsilon \tilde{D}^{ \pm}, \quad D^{ \pm}-\{Q\}=\tilde{D}^{ \pm}-\left\{H_{2}\right\} \text { and } \mathcal{N}-\left\{B_{a}^{\prime \prime \prime}\right\}=\tilde{\mathcal{N}}-\left\{\tilde{B}_{e}^{\prime}\right\},
$$

where $H_{1}=B_{a}^{\prime} " \cup Q+a_{\rho}$ and $H_{2}=\left(B_{a}^{\prime} "-V\left(\tilde{B}_{e}^{\prime}\right)\right) \cup Q+a_{\rho}$.

Proof: We only consider the case when an $\mathcal{N}$-surrounded graph $Q \varepsilon D^{+}$, 
$A\left(T_{r}^{\prime}\right)=A^{-}\left(T_{r}^{\prime}\right)$ and $\partial^{+} a_{\rho}=y$. Let $B_{a}^{\prime \prime}$ be the leaf branch as to some $a^{\prime \prime}$ and $T_{r}^{\prime}$ such that $B_{a}^{\prime}$ " equals a surrounding $\mathcal{N}$-graph of $Q$, and define $X$ by $X=$ $\left\{a \varepsilon A^{+}\left(\tilde{T}_{r}^{\prime}\right) \cap A\left(\tilde{P}_{v y}\right): x(a)=b(a)\right\}$ where $x(a)$ is the value given in Step (5.iii). By JOIN-operations, if $X=\phi$, then Case (1) is obtained. Otherwise we have

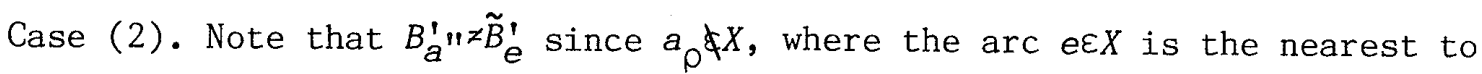
vertex w.

We prove the next proposition, based on Propositions 4.1 and 4.2 .

Proposition 4.3. Steps (5.iii) (5.v) in Trimming operation are repeated at most $2|V|-4$ times.

Proof: Define $p, \tilde{p}, q$ and $\tilde{q}$ as follows.

$p=\Sigma\{|V(Q)|: Q \varepsilon \mathcal{N}\}, q=|\mathcal{N}|, \tilde{p}=\Sigma\{|V(\tilde{Q})|: \tilde{Q} \varepsilon \tilde{\mathcal{N}}\}$ and $\tilde{q}=|\tilde{\mathcal{N}}|$.

We only prove the case where an $\mathcal{N}$-surrounded graph $Q$ is in $\mathscr{D}^{+}$. After one pivot operation, from Propositions 4.1 and 4.2 we have $p=\tilde{p}, q-1=\tilde{q}$ for Case $C 1$, while Case C2 shows $p>\tilde{p}, q \geqq \tilde{q}$. It is easy to see $|V|-2 \geqq p \geqq q$, and that $p$ or $q$ does not increase after each pivot operation. If the value $p+q$ vanishes, then Trimming operation is over. So Steps (5.iii) (5.v) are repeated at most $2|V|-4$ pivot operations.

\subsubsection{Pivot Operations except Trimming Operations}

The next proposition can be proved similarly as Proposition 3.1.

Proposition 4.4. For the leaf branch $B_{a} * \varepsilon D^{ \pm}$of $T_{r}$ chosen in Step 3, we have an $\operatorname{arc} a_{\rho}$ and the circulation $x$ such that

$$
x\left(a_{\rho}\right)=\left\{\begin{array}{cc}
0 & \left(\partial^{ \pm} a_{\rho} \varepsilon V\left(B_{a^{*}}\right),\right. \\
b\left(a_{\rho}\right) & \left(\partial^{\mp} a_{\rho} \varepsilon V\left(B_{a^{*}}\right)\right) \cdot \square
\end{array}\right.
$$

After doing a pivot operation except Trimming operations, the circulation changes on the cycle formed by $T_{r}$ and $a_{\rho}$. For $V^{\prime}, v^{\prime \prime} \varepsilon V$, let $P_{V^{\prime}} v^{\prime \prime}$ ( $\tilde{P}_{V^{\prime} V^{\prime \prime}}$ ) be the path of $T_{r}\left(\tilde{T}_{r}\right)$, respectively. Assume that vertex $V^{*}($ $\left.V^{*} \varepsilon V\left(P_{r u}\right) \cap V\left(P_{r y}\right)\right)$ is the farthest from root $r$ on $P_{r y}$. (See Fig.9.) Then the following proposition is easily shown.

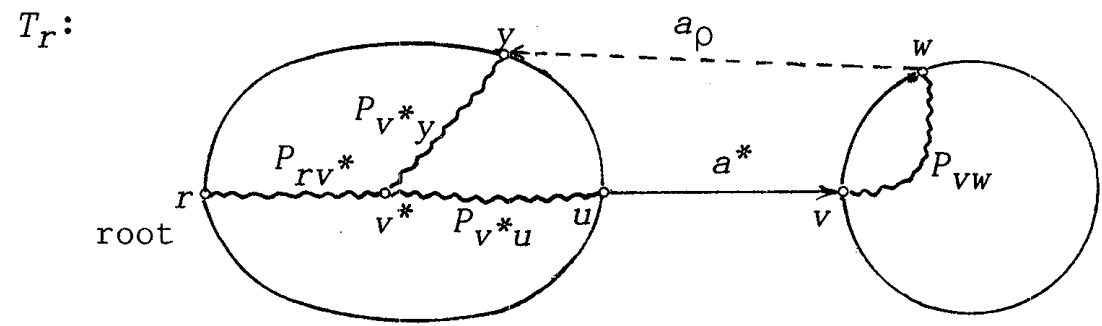

Fig.9 
Proposition 4.5. $\left\{Q^{\prime} \varepsilon \underline{D}^{ \pm} \cup \underline{\mathcal{N}}: V\left(Q^{\prime}\right) \cap V^{*}=\phi\right\}=\left\{\check{Q} \varepsilon \underline{\tilde{D}}^{ \pm} \cup \tilde{\tilde{\mathcal{N}}}: V(\tilde{Q}) \cap \tilde{V}^{*}=\phi\right\}$, where $V^{*}=V\left(P_{r w}\right) \cup V\left(P_{V^{*}}\right)$ and $\tilde{V}^{*}=V\left(\tilde{P}_{r V}\right) \cup V\left(\tilde{P}_{V^{*} u}\right)$.

From Proposition 4.5, consider the graph obtained by removing $Q^{\prime} \varepsilon \&$ such that $V\left(Q^{\prime}\right) \cap V^{*}=\phi$, where $\mathcal{\&}=\underline{D}^{+} \cup \underline{\mathcal{D}} \cup \underline{\mathcal{N}}$. That is, let us execute the next operation CUT $\left(T_{r}, a^{*}, a_{\rho}, V^{*}\right.$ ) ( or simply CUT-operation) by using the circulation $x$ obtained in Section 2.1 and $z_{r}=0$.

$\operatorname{CUT}\left(T_{r}, a^{*}, a_{\rho}, V^{*}\right):$

While there is a leaf branch $B_{a^{\prime}} \varepsilon \&$ of $T_{r}$ such that $V\left(B_{a^{\prime}}\right) \cap V^{*}=\phi$ for some $a^{\prime}$, then carry out $\operatorname{ADJUST}\left(T_{r}, a^{\prime},\left(x(a): a \varepsilon A\left(T_{r}\right)\right), z_{r}\right)$ and set $\mathcal{\&}=\mathcal{\&}-\left\{B_{a^{\prime}}\right\}$. Otherwise, we stop this operation.

When we apply CUT-operation to both $T_{r}$ and $\tilde{T}_{r}$, we finally have two some rooted subtrees and renewed values, i.e., let

(4.1) $T_{r}^{0},\left(x^{0}(a): a \varepsilon A\left(T_{r}^{0}\right)\right)$ and $z_{r}^{0}$ be the rooted subtree and the values determined after doing $\operatorname{CUT}\left(T_{r}, a *, a_{\rho}, V^{*}\right)$,

(4.2) $\tilde{T}_{r}^{0},\left(\tilde{X}^{0}(a): a \varepsilon A\left(\tilde{T}_{r}^{0}\right)\right)$ and $\tilde{z}_{r}^{0}$ be the rooted subtree and the values determined after doing $\operatorname{CUT}\left(\tilde{T}_{r}, a^{*}, a_{\rho}, \tilde{V}^{*}\right)$.

Now, we are ready to observe the change of the classes and the deficiencies $d(D), d(\tilde{D})$ between the new trees $T_{r}^{0}$ and $\tilde{T}_{r}^{0}$, in place of $T_{r}$ and $\tilde{T}_{r}$. For the leaf branch $B_{a^{*} \varepsilon \mathscr{D}^{ \pm}}^{0}$ of $T_{r}^{0}$, let $B_{i}^{0} \subset B_{a^{*}}^{0}{ }^{\prime}(1 \leqq i \leqq k)$ be the members of $\mathcal{D}^{ \pm}$such that these $B_{i}^{0}$ are ordered in such a way that $w \varepsilon V\left(B_{1}^{0}\right)$ and that $B_{i+1}^{0}$ appears after $B_{i}^{0}$ toward root $r$. ( $a^{*}$ is the arc chosen in (3.i) of Step 3 in Section 3.3.2.) Let $a_{i} \varepsilon A\left(T_{r}^{0}\right)$ be the arc joined by $B_{i}^{0}$ and $B_{i+1}^{0}$, where we define $a_{k}=a^{*}$. For each $a_{i}$ in $B_{a^{*}}^{0}$ of $D^{ \pm}$and the value $x^{0}\left(a_{i}\right)$ defined in (4.1), let

$$
\Delta_{i}= \begin{cases}-x^{0}\left(a_{i}\right) & \text { if } a_{i} \varepsilon A^{ \pm}\left(T_{r}^{0}\right), \\ x^{0}\left(a_{i}\right)-b\left(a_{i}\right) & \text { if } a_{i} \varepsilon A^{\mp}\left(T_{r}^{0}\right),\end{cases}
$$

and $\Delta_{0}=0$. Then the following proposition, which is easily proved, characterizes each $B_{i}^{0}$.

Proposition 4.6. We have $B_{i}^{0} \varepsilon \underline{D}^{ \pm}(1 \leqq i \leqq k)$ for the leaf branch $B_{a}^{0} \approx \mathcal{D}^{ \pm}$of $T_{r}^{0}$ if and only if the next conditions are satisfied.

$$
\begin{array}{ll}
x^{0}\left(a_{i}\right)+\Delta_{i-1}<0 & \text { if } a_{i} \varepsilon A^{ \pm}\left(T_{r}^{0}\right), \\
x^{0}\left(a_{i}\right)-\Delta_{i-1}>b\left(a_{i}\right) & \text { if } a_{i} \varepsilon A^{\mp}\left(T_{r}^{0}\right), \\
0 \leqq x^{0}(a)+\Delta_{i-1}<b(a) & \text { if } a \varepsilon A^{ \pm}\left(T_{r}^{0}\right) \cap A\left(B_{i}^{0}\right) \cap A\left(P_{V W}^{0}\right), \\
0<x^{0}(a)-\Delta_{i-1} \leqq b(a) & \text { if } a \varepsilon A^{\mp}\left(T_{r}^{0}\right) \cap A\left(B_{i}^{0}\right) \cap A\left(P_{V W}^{0}\right), \\
0 \leqq x^{0}(a)<b(a) & \text { if } a \varepsilon A^{ \pm}\left(T_{r}^{0}\right) \cap A\left(B_{i}^{0}\right)-A\left(P_{V W}^{0}\right), \\
0<x^{0}(a) \leqq b(a) & \text { if } a \varepsilon A^{\mp}\left(T_{r}^{0}\right) \cap A\left(B_{i}^{0}\right)-A\left(P_{V W}^{0}\right),
\end{array}
$$


where $P_{v w}^{0}$ is the path of $T_{r}^{0}$ and $x^{0}(a)\left(\operatorname{acA}\left(T_{r}^{0}\right)\right)$ appearing above are the values defined in (4.1). Moreover we have $\tilde{d}\left(B_{i}^{0}\right)=\Delta_{i}-\Delta_{i-1}>0$ and $d\left(B_{a}^{0}\right)=\Delta_{k} \cdot \square$

The following proposition, which is easily proved, shows the relation between $\left(x^{0}(a): a \varepsilon A\left(B_{a}^{0}\right)\right)$ and $\left(\tilde{x}^{0}(a): a \varepsilon A\left(\tilde{B}_{a_{\rho}}^{0}\right)\right)$ defined in (4.1) and (4.2), where $B_{a^{*}}^{0}\left(\tilde{B}_{a_{\rho}}^{0}\right)$ is the leaf branch as to $T_{r}^{0}$ and $\operatorname{arc} a^{*}\left(\tilde{T}_{r}^{0}\right.$ and arc $\left.a_{\rho}\right)$.

Proposition 4.7. For the leaf branch $B_{a}^{0} \approx \varepsilon D^{ \pm}$of $T_{r}^{0}$, we have

$$
\begin{aligned}
& \tilde{x}^{0}(a)=x^{0}(a)-\Delta_{k} \quad \text { if } a \varepsilon A^{ \pm}\left(\tilde{T}_{r}^{0}\right) \cap A\left(\tilde{B}_{a_{\rho}}^{0}\right) \cap A\left(\tilde{P}_{V y}^{0}\right) \text {, } \\
& \tilde{x}^{0}(a)=x^{0}(a)+\Delta_{k} \quad \text { if } a \varepsilon A^{\mp}\left(\tilde{T}_{r}^{0}\right) \cap A\left(\tilde{B}_{a_{\rho}}^{0}\right) \cap A\left(\tilde{P}_{v y}^{0}\right) \text {, } \\
& \text { (2) } \quad \tilde{x}^{0}(a)<b(a) \\
& \text { if } \operatorname{a\varepsilon A^{\pm }}\left(\tilde{T}_{r}^{0}\right) \cap A\left(\tilde{B}_{a_{\rho}}^{0}\right) \text {, } \\
& \tilde{x}^{0}(a)>0
\end{aligned}
$$

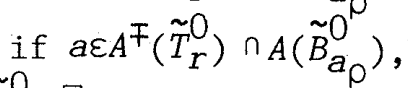

where $\tilde{P}_{v y}^{0}$ denotes the path of $\tilde{T}_{r}^{0}$.

First, we consider how the leaf branch $\tilde{B}_{a_{\rho}}^{0}$ of $\tilde{T}_{r}^{0}$ is partitioned by Tree partitioning algorithm. For leaf branch $B_{a^{*}}^{0}$ in $D^{ \pm}$of $T_{r}^{0}$, let

$$
\text { (4.4) } \quad U^{ \pm}=\left\{a \varepsilon A^{\mp}\left(\tilde{T}_{r}^{0}\right) \cap A\left(\tilde{P}_{v y}^{0}\right): \tilde{x}^{0}(a)>b(a)\right\} \cup\left\{a \varepsilon A^{ \pm}\left(\tilde{T}_{r}^{0}\right) \cap A\left(\tilde{P}_{v y}^{0}\right): \tilde{x}^{0}(a)<0\right\} \text {, }
$$
where $\tilde{x}^{0}(a)\left(a \varepsilon A\left(\tilde{T}_{r}^{0}\right)\right)$ is the value defined in $(4.2)$. We assume that $U^{ \pm}$is not empty in the future discussion. Let $a^{1} \varepsilon U^{ \pm}$be the arc which is the nearest to vertex $v$ on the path $\tilde{P}_{v y}^{0}$ of $\tilde{T}_{r}^{0}$, and

$$
\Delta_{a} 1=\left\{\begin{array}{cc}
b\left(a^{1}\right)-x^{0}\left(a^{1}\right) & \text { if } a^{1} \varepsilon A^{\mp}\left(\tilde{T}_{r}^{0}\right), \\
x^{0}\left(a^{1}\right) & \text { if } a^{1} \varepsilon A^{ \pm}\left(\tilde{T}_{r}^{0}\right),
\end{array}\right.
$$

where the value $x^{0}\left(a^{1}\right)$ is defined in (4.1). Then we see that the leaf branch $\tilde{B}_{a}^{0} 1$ of $\tilde{T}_{r}^{0}$ is in $\underline{\mathscr{D}}^{ \pm}$at (P2). After doing ADJUST-operation in (P4), we have the values $\tilde{x}^{1}(a)\left(a \varepsilon A\left(\tilde{T}_{r}^{1}\right)\right)$ for $\tilde{T}_{r}^{1} \tilde{T}_{r}^{0}-V\left(\tilde{B}_{a}^{0} 1\right)$ such that the following proposition shows.

Proposition 4.8. For the leaf branch $B_{a^{* \varepsilon}}^{0} D^{ \pm}$of $T_{r}^{0}$ and nonempty set $U^{ \pm}$, we have the following (1), (2) and (3).

(1) $\Delta_{k}>\Delta_{a} 1>0$.

$$
\begin{array}{ll}
\tilde{x}^{1}(a)=x^{0}(a)-\Delta_{a} 1 & \text { if } a \varepsilon A^{ \pm}\left(\tilde{T}_{r}^{1}\right) \cap A\left(\tilde{B}_{a_{\rho}}^{0}\right) \cap A\left(\tilde{P}_{v y}^{1}\right), \\
\tilde{x}^{1}(a)=x^{0}(a)+\Delta_{a} 1 & \text { if } a \varepsilon A^{\mp}\left(\tilde{T}_{r}^{1}\right) \cap A\left(\tilde{B}_{a_{\rho}}^{0}\right) \cap A\left(\tilde{P}_{v y}^{1}\right), \\
\tilde{x}^{1}(a)<b(a) & \text { if } a \varepsilon A^{ \pm}\left(\tilde{T}_{r}^{1}\right) \cap A\left(\tilde{B}_{a_{\rho}}^{0}\right), \\
\tilde{x}^{1}(a)>0 & \text { if } a \varepsilon A^{\mp}\left(\tilde{T}_{r}^{1}\right) \cap A\left(\tilde{B}_{a_{\rho}}^{0}\right),
\end{array}
$$$$
\text { (3) } \quad \tilde{x}^{1}(a)<b(a)
$$

where $\tilde{P}_{v y}^{1}$ is the path of $\tilde{T}_{r}^{1}$ and $x^{0}(a)\left(a \varepsilon A\left(\tilde{T}_{r}^{1}\right)\right)$ appearing above is the value defined in (4.1).

Proof: We only consider the case when $B_{a^{* \varepsilon \varepsilon} \mathscr{D}^{+}}$and $A\left(T_{r}^{0}\right)=A^{+}\left(T_{r}^{0}\right)$. We check Case (2) first. From $a^{1} \varepsilon U^{+}$, Propositions 4.6 and 4.7 , we have $\Delta_{k}>\Delta_{a} 1$. Let $\tilde{x}^{1}(a)\left(\operatorname{a\varepsilon A}\left(\tilde{T}_{r}^{1}\right)\right)$ be the values obtained by $\operatorname{ADJUST}\left(\tilde{T}_{r}^{0}, a^{1},\left(\tilde{x}^{0}(a): a \varepsilon A\left(\tilde{T}_{r}^{0}\right)\right)\right.$, 
$\left.\tilde{z}_{r}^{0}\right)$ where $\tilde{T}_{r}^{1}=\tilde{T}_{r}^{0}-V\left(\tilde{B}_{a}^{0} 1\right)$ for the leaf branch $\tilde{B}_{a}^{0} 1$ of $\tilde{T}_{r}^{0}$. Then from Proposition 4.7 we have

$$
\tilde{x}^{1}(a)=\tilde{x}^{0}(a)-\left(\Delta_{k}-\Delta_{a} 1\right)=x^{0}(a)+\Delta_{a} 1 \quad\left(a \varepsilon A\left(\tilde{P}_{v y}^{1}\right) \cap A\left(\tilde{B}_{a}^{0}\right)\right),
$$

where $\tilde{x}^{0}(a)$ (resp. $x^{0}(a)$ ) is the value defined in (4.2) (resp. (4.1)) and $\tilde{P}_{v y}^{1}$ is the path of $\tilde{T}_{r}^{1}$. When we deal with (3), it suffices to see the arcs of $\tilde{P}_{v y}^{1}$. The following three cases can be considered with respect to $a^{1}$ :

Case $A: a^{1}=a \rho$,

Case $B: a^{1}=a_{j}$ for some $a_{j}$ joining $B_{j}^{0}$ and $B_{j+1}^{0}$,

Case C: $a^{1} \varepsilon A\left(B_{j}^{0}\right) \cap A\left(\tilde{P}_{v W}^{0}\right)$ for some $j$ and the path $\tilde{P}_{v W}^{0}$ of $\tilde{T}_{r}^{0}$.

We examine Case $B$ only. From Proposition 4.6 and $\Delta_{a} 1<\Delta_{k}$, we have Case (1) of

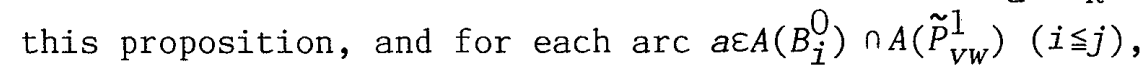

$$
\tilde{x}^{1}(a)=x^{0}(a)+\Delta_{a} 1 \geqq x^{0}(a)+\Delta_{i}>x^{0}(a)+\Delta_{i-1} \geqq 0,
$$

where $x^{0}(a), \Delta_{i}$ are defined in $(4.1),(4.3)$, respectively. Similarly, we have $\tilde{x}^{1}\left(a_{i}\right)>0$ for arc $a_{i}(i \leqq j-1)$ joining $B_{i}^{0}$ and $B_{i+1}^{0}$.

The following proposition is the result of Tree Partitioning for the leaf branch $\tilde{B}_{a_{\rho}}^{0}$ of $\tilde{T}_{r}^{0}$. Let $a^{i}$ be the arc for which Tree Partitioning is implemented at the $i$-th repetition.

Proposition 4.9. If Tree Partitioning is executed for $\tilde{B}_{a_{\rho}}^{0}$, then we have the following (1) or (2) for some integer $n$ and the leaf branch $B_{a}^{0} * \varepsilon D^{ \pm}$of $T_{r}^{0}$.

(1) $\quad \tilde{B}_{a}^{0} 1 \varepsilon \underline{\partial ू}^{ \pm}$,

(2) $\tilde{B}_{a}^{0} i+1-V\left(\tilde{B}_{a}^{0} \dot{i}\right) \varepsilon \underline{\mathscr{D}}^{ \pm} \quad(1 \leqq i \leqq n-1)$,

where $\tilde{B}_{a}^{0} \cdot(1 \leqq i \leqq n)$ are leaf branches of $\tilde{T}_{r}^{0}$.

Proof: We have already checked Case (1). Concerning Case (2), we apply Propositions 4.7 and 4.8 repeatedly.

After ( $\mathrm{P} 4)$ of Tree partitioning algorithm has been done by the arc $a^{n} \varepsilon A\left(\tilde{B}_{a_{\rho}}^{0}\right)$ chosen finally in (P2) with respect to $\tilde{B}_{a_{\rho}}^{0}$, do the algorithm on the path between $w$ and $v^{*}\left(u\right.$ and $v^{*}$ ) of $T_{r}^{0}$ ( the current rooted subtree of $\left.\tilde{T}_{r}^{0}\right)$, respectively. That is, let

(4.5) $\quad \tilde{T}_{r}^{n}=\tilde{T}_{r}^{0}-V\left(\tilde{B}_{a^{n}}^{0}\right)-\cup\left\{V(\tilde{Q}): \tilde{Q} \varepsilon \underline{\tilde{D}}^{+} \cup \underline{\mathscr{D}}^{-} \cup \underline{\tilde{N}}, V\left(\tilde{P}_{u v^{*}}^{0}\right) \cap V(\tilde{Q})=\phi\right\}$,

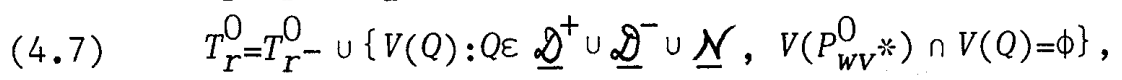

where $\tilde{P}_{u v^{*}}^{0}\left(P_{w V^{*}}^{0}\right)$ is the path of $\tilde{T}_{r}^{0}$ ( the old $T_{r}^{0}$ ), respectively and $\tilde{B}_{a^{n}}^{0}$

is the leaf branch of $\tilde{T}_{r}^{0}$.

For the leaf branch $B_{a}^{0 * \varepsilon} D^{ \pm}$of the old $T_{r}^{0}$, let

$$
\Delta_{a^{n}}=\left\{\begin{array}{cc}
b\left(a^{n}\right)-x^{0}\left(a^{n}\right) & \left(a^{n} \varepsilon A^{\mp}\left(\tilde{T}_{r}^{0}\right)\right), \\
x^{0}\left(a^{n}\right) & \left(a^{n} \varepsilon A^{ \pm}\left(\tilde{T}_{r}^{0}\right)\right),
\end{array}\right.
$$


where $x^{0}\left(a^{n}\right)$ is the value defined in (4.1). We assume that

(4.9) $\quad x^{0}(a)\left(a \varepsilon A\left(T_{r}^{0}\right)\right)$ and $z_{r}^{0}$ are the values determined by the repetition of (P4) until the new $T_{r}^{0}$ is obtained,

(4.10) $\tilde{x}^{n}(a)\left(a \varepsilon A\left(\tilde{T}_{r}^{n}\right)\right)$ and $\tilde{z}_{r}^{n}$ are the values determined by the repetition of (P4) until $\tilde{T}_{r}^{n}$ is obtained.

Note that we compare the new $T_{r}^{0}$ with $\tilde{T}_{r}^{n}$ from now on and that for the new $T_{r}^{0}$, the values $\left(x^{0}(a): a \varepsilon A\left(T_{r}^{0}\right)\right)$ in $(4.1)$ differ from those in (4.9) on the path of the new $T_{r}^{0}$ between $v^{*}$ and $r$.

We continue Tree Partitioning for $Q^{\prime} \varepsilon D \cup \mathcal{N}$ such that $y \varepsilon V\left(Q^{\prime}\right)$. If $Q^{\prime}$ is in $\mathscr{D}^{+}\left(\operatorname{resp} . \mathcal{D}^{-}\right)$, then let $B_{i}^{\prime} \subset Q^{\prime}(1 \leqq i \leqq m)$ be the members of $\underline{D}^{+}$(resp. $\underline{D}^{-}$) such that they are ordered so that $B_{i+1}^{\prime}$ appears after $B_{i}^{\prime}$ toward root $r$ ( we see that $y \varepsilon V\left(B_{1}^{\prime}\right)$. ). We denote by $a_{i}^{\prime}$ the arc connecting $B_{i+1}^{\prime}$ to $B_{i}^{\prime}$, where $a_{m}^{\prime}=a^{\prime}$ for some leaf branch $B_{a}^{0}{ }^{\prime}=Q^{\prime}$ of $T_{r}^{0}$. Similarly, we also define $B_{i}^{\prime}$ and $a_{i}^{\prime}$ for $Q^{\prime} \varepsilon \mathcal{N}$. In the example of Fig.10, we have $m=3$ and $Q^{\prime}=B_{a}^{0} \cdot$. In this figure, $Q^{\prime}$ is composed of subtrees $B_{i}^{\prime}(1 \leqq i \leqq 3)$ of $Q^{\prime}$ and two tree $\operatorname{arcs} a_{1}^{\prime}, a_{2}^{\prime}$.

$\tilde{T}_{r}^{n}:$

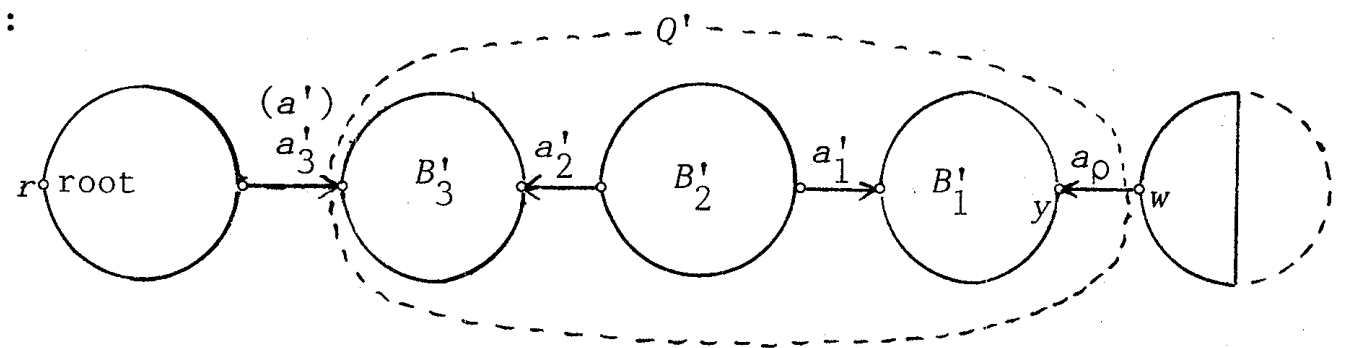

Fig.10

First assuming $Q^{\prime} \varepsilon D^{ \pm}$as to the leaf branch $B_{a}^{O * \varepsilon D^{ \pm}}$of the rooted subtree in (4.1), we have the following proposition.

Proposition 4.10. If $B_{a^{*} \varepsilon D^{ \pm}}$and $Q^{\prime} \varepsilon D^{ \pm}$, then we have

(1) $B_{a^{*}}^{0} \cup Q^{\prime}+a \rho \varepsilon \tilde{D}^{ \pm}$,

(2) $\quad D^{ \pm}-\left\{B_{a^{*}}^{0}, Q^{\prime}\right\}=\tilde{D}^{ \pm}-\left\{B_{a^{*} \cup Q^{\prime}+a_{\rho}}^{0}, \quad D^{\mp}=\tilde{D}^{\mp}\right.$.

Proof: We only check the case where $B_{a^{*}}^{0} \mathcal{D}^{+}$and $A\left(T_{r}^{0}\right)=A^{-}\left(T_{r}^{0}\right)$. Let $\left(x^{0}(a): a \varepsilon A\left(T_{r}^{0}\right)\right), z_{r}^{0}$ (resp. $\left.\left(\tilde{x}^{n}(a): a \varepsilon A\left(\tilde{T}_{r}^{n}\right)\right), \tilde{z}_{r}^{n}\right)$ be the values defined in (4.9) (resp. (4.10)). If $B_{1}^{\prime}\left(B_{1}^{\prime} \subset Q^{\prime}\right)$ equals some rooted subtree $T_{r}^{\prime}$, then from $B_{1}^{\prime} \varepsilon \underline{\mathscr{D}}^{+}$we have

$$
\tilde{z}_{r}^{n}=z_{r}^{0}-\Delta_{a} n<0 \text {. }
$$

Note that we have $z_{r}^{0}<0$ in (4.11). Otherwise, from $x^{0}\left(a_{1}^{\prime}\right)>b\left(a_{1}^{\prime}\right)$ and Proposition 4.6 , it follows that

$$
\tilde{x}^{n}\left(a_{1}^{\prime}\right)=x^{0}\left(a_{1}^{\prime}\right)+\Delta_{a} n>b\left(a_{1}^{\prime}\right) \text {. }
$$


Note that we have the arc $a_{1}^{\prime}$ and $\tilde{x}^{n}(a)=x^{0}(a)+\Delta_{a} n>0\left(a \varepsilon A\left(B_{1}^{\prime}\right) \cap A\left(\tilde{P}_{y r}^{n}\right)\right)$ for the path $\tilde{P}_{y r}^{n}$ of $\tilde{T}_{r}^{n}$. Let $X=\left\{a \varepsilon A\left(B_{1}^{\prime}\right) \cap A\left(\tilde{P}_{y r}^{n}\right): \tilde{x}^{n}(a)>b(a)\right\}$. If $X$ is empty, then we easily have (1) and (2) of this proposition. Let $e^{1} \varepsilon X$ be the arc which is the nearest to vertex $y$. In (P2) of Tree partitioning algorithm, we have (4.13) $\quad \tilde{B}_{e}^{n} 1 \varepsilon \underline{\mathscr{D}}^{+}$, where $\tilde{B}_{e}^{n} 1$ is the leaf branch of $\tilde{T}_{r}^{n}$. Define $\Delta_{e} 1$ by replacing $a^{n}$ with $e^{1}$ in (4.8), and we have $\Delta_{e} 1 \geqslant 0$ from Proposition 4.6. If $\Delta_{e} 1=0$, then we have $B_{1}^{\prime}-V\left(\tilde{B}_{e}^{n} 1\right) \varepsilon \tilde{D}^{+}$and that other members in the classes of $\tilde{T}_{r}^{n}$ are the same as those of $T_{r}^{0}$. If $\Delta_{e} 1>0$, then we see that every time (P2) is executed, a member $D$ of $\tilde{D}^{+}$is derived and that there exist no members of $\tilde{\mathcal{N}}$ obtained from $D$. Finally, we have this proposition by JOIN-operations.

Second, assume that $Q^{\prime}$ is in $\mathcal{N}$ with respect to the leaf branch $B_{a}^{0} \approx \varepsilon D^{ \pm}$ of ( the old ) $T_{\Gamma}^{0}$. We see that $Q^{\prime}$ equals either the rooted new tree $T_{r}^{0}$ or some leaf branch $B_{a}^{0}$ ' of ( the new ) $T_{r}^{0}$. If $Q^{\prime}$ equals $T_{r}^{0}$, then we have the following proposition.

Proposition 4.11. For $B_{a}^{0} \approx \varepsilon D^{ \pm}$and $Q^{\prime}=T_{r}^{0} \varepsilon \mathcal{N}$, we have either (1) or (2):

(1) For some rooted subtree $T_{r}^{3}$ of $T_{r}^{0}$ such that $P_{y r}^{0} \subset T_{r}^{3}$, we have

$$
B_{a^{*}}^{0} \cup T_{r}^{3}+a \rho \varepsilon \tilde{D}^{ \pm}, \quad D^{ \pm}-\left\{B_{a}^{0}\right\}=\tilde{D}^{ \pm}-\left\{B_{a}^{0} \cup T_{r}^{3}+a_{\rho}\right\}, \quad D^{\mp}=\tilde{D}^{\mp} .
$$

(2) For some leaf branch $B_{e}^{0 *}$ and some subtree $Q^{0} \subset B_{e^{*}}^{0}$ of $T_{r}^{0}$ such that

$V\left(P_{\mathrm{yr}}^{0}\right) \cap V\left(B_{e^{*}}^{0}\right) \subset V\left(Q^{0}\right)$, we have

$$
B_{a}^{0} \cup Q^{0}+a_{\rho} \varepsilon \tilde{D}^{ \pm}, \quad D^{ \pm}-\left\{B_{a}^{0}\right\}=\tilde{D}^{ \pm}-\left\{B_{a}^{0} \cup Q^{0}+a_{\rho}\right\}, \quad D^{\mp}=\mathscr{D}^{\mp},
$$

where $P_{y r}^{0}$ is the path of $T_{r}^{0}$.

Proof: We only prove the case where $B_{a}^{0} * \varepsilon D^{+}$and $A\left(T_{r}^{0}\right)=A^{-}\left(T_{r}^{0}\right)$. Let $\left(x^{0}(a): a \varepsilon A\left(T_{r}^{0}\right)\right)$ and $z_{r}^{0},\left(\tilde{x}^{n}(a): a \wedge_{A}\left(\tilde{T}_{r}^{n}\right)\right)$ and $\tilde{z}_{r}^{n}$ be the values defined in (4.9), (4.10), respectively. From $Q^{\prime}=T_{r}^{0} \varepsilon \mathcal{N}$, it follows that

(4.14) $\quad \tilde{x}^{n}(a)=x^{0}(a)+\Delta_{a} n>0 \quad\left(a \varepsilon A\left(\tilde{P}_{y r}^{n}\right)\right)$,

(4.15) $\quad \tilde{z}_{r}^{n}=z_{r}^{0}-\Delta_{a}^{n<0}$,

where $\tilde{P}_{y r}^{n}$ is the path of $\tilde{T}_{r}^{n}$. Note here that we have $0 \leqq x^{0}(a) \leqq b(a)\left(a \varepsilon A^{-}\left(T_{r}^{0}\right)\right)$ and $z_{r}^{0}=0$. Define $X=\left\{a \varepsilon A\left(\tilde{P}_{y r}^{n}\right): \tilde{x}^{n}(a)>b(a)\right\}$. For $X=\phi$, we have (1) of this proposition. If $X \not{\not} \phi$, then employ the same argument as in Proposition 4.10.

Assume that $Q^{\prime} \varepsilon \mathcal{N}$ equals some leaf branch $B_{a}^{0}$, of ( the new ) $T_{r}^{0}$, then we have a member $Q^{\prime \prime} \varepsilon \mathcal{D}^{+} \cup D^{-}$such that $Q^{\prime}$ and $Q^{\prime \prime}$ are joined by the arc $a^{\prime}$. Here, if $Q^{\prime \prime}$ belongs to $D^{\mp}$ for the leaf branch $B_{a}^{0} \mathcal{F}^{ \pm} D^{ \pm}$of ( the old ) $T_{r}^{0}$, then we have the following proposition which can be shown in the same way as Proposition 4.11。

Proposition 4.12. If $Q^{\prime}=B_{a}^{0}, \varepsilon \mathcal{N}$ and $Q^{\prime \prime} \varepsilon D^{\mp}$ for $B_{a^{*}}^{0} \varepsilon D^{ \pm}$, then we have: 
For some subtree $Q^{3} \subset B_{1}^{\prime}$ of $Q^{\prime}$ such that $V\left(\tilde{P}_{y r}^{n}\right) \cap V\left(B_{1}^{\prime}\right) \subset V\left(Q^{3}\right)$,

$$
B_{a}^{0} \cup Q^{3}+a \rho \varepsilon \tilde{D}^{ \pm}, \quad D^{ \pm}-\left\{B_{a}^{0}\right\}=\tilde{D}^{ \pm}-\left\{B_{a^{*}}^{0} \cup Q^{3}+a_{\rho}\right\}, \quad D^{\mp}=\tilde{D}^{\mp},
$$

where $\tilde{P}_{y r}^{n}$ is the path of $\tilde{T}_{r}^{n}$.

On the other hand, if $Q^{\prime}=B_{a}^{0}, \mathcal{N}$ and $Q^{\prime \prime}$ joined by the arc $a^{\prime}$ is in $D^{ \pm}$ as to the leaf branch $B_{a^{*} \varepsilon D^{ \pm}}^{0}$, then examine the graph $Q^{\prime \prime}$ in detail. For $Q^{\prime \prime} \varepsilon D^{ \pm}$, let $B_{i}^{\prime \prime} \subset Q^{\prime \prime}\left(1 \leqq i \leqq k^{\prime \prime}\right)$ be the members of $\underline{D}^{ \pm}$such that $B_{i+1}^{\prime \prime}$ comes after $B_{i}^{\prime \prime}$ toward root $r$. In the same way as $a_{i}^{\prime}$ is defined, denote by $a_{i}^{\prime \prime}$ the arc joining $B_{i}^{\prime \prime}$ and $B_{i+1}^{\prime \prime}$. Then we have:

Proposition 4.13. If $Q^{\prime} \varepsilon \mathcal{N}$ equals the leaf branch $B_{a}^{0}$, and $Q^{\prime \prime} \varepsilon \mathcal{D}^{ \pm}$for the leaf branch $B_{a}^{0} * \varepsilon D^{ \pm}$, then we have either (1) or (2).

(1) $H_{3} \varepsilon \tilde{D}^{ \pm}, \quad D^{ \pm}-\left\{B_{a^{*}}^{O}, Q^{\prime \prime}\right\}=\tilde{D}^{ \pm}-\left\{H_{3}\right\}, \quad D^{\mp}=\tilde{D}^{\mp}$,

(2) For some leaf branch $B_{e}^{4}$, of $B_{a}^{0}$, ,

$$
H_{4} \varepsilon \tilde{D}^{ \pm}, \quad D^{ \pm}-\left\{B_{a^{*}}^{O}, Q^{\prime \prime}\right\}=\tilde{D}^{ \pm}-\left\{H_{4}\right\}, \quad D^{\mp}=\tilde{D}^{\mp},
$$

where $H_{3}=\left(B_{a^{*}+a_{\rho}}^{0}\right) \cup\left(B_{a}^{0}+a^{\prime}\right) \cup Q^{\prime \prime}$ and $H_{4}=B_{a^{*}}^{0} \cup B_{e^{\prime}+a_{\rho}}^{4}$.

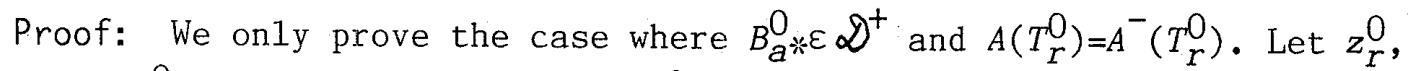
$\left(x^{0}(a): a \varepsilon A\left(T_{r}^{0}\right)\right)\left(\operatorname{resp} . \tilde{z}_{r}^{n},\left(\tilde{x}^{n}(a): a \varepsilon A\left(\tilde{T}_{r}^{n}\right)\right)\right)$ be the values defined in (4.9) (resp. (4.10)). From $B_{a}^{0}, \varepsilon \mathcal{N}$ and $B_{1}^{\prime \prime} \subset Q^{\prime \prime} \varepsilon D^{+}$, we have

$$
\tilde{z}_{r}^{n}<0
$$$$
\left(B_{1}^{\prime \prime}=T_{r}^{0}-V\left(Q^{\prime}\right)\right) \text {, }
$$$$
\tilde{x}^{n}\left(a_{1}^{\prime \prime}\right)>b\left(a_{1}^{\prime \prime}\right)
$$
( otherwise).

Note that we have the arc $a_{1}^{\prime \prime}$ if $B_{1}^{\prime \prime}$ is not equal to $T_{r}^{0}-V\left(Q^{\prime}\right)$. For the path $\tilde{P}_{y r}^{n}$ of $\tilde{T}_{r}^{n}$, let $X=\left\{a \varepsilon A\left(B_{a}^{0}, \cup B_{1}^{\prime \prime}+a^{\prime}\right) \cap A\left(\tilde{P}_{y r}^{n}\right): \tilde{x}^{n}(a)>b(a)\right\}$. We have (1) of this proposition for $X=\phi$, while we apply the same argument as in Proposition 4.10 for nonempty set $X$. $\square$

Finally suppose that $Q^{\prime}$ is in $\mathcal{D F}^{F}$ for the leaf branch $B_{a}^{O} \approx \varepsilon D^{ \pm}$of ( the old ) $T_{r}^{0}$ where it will be only shown that the deficiency $d(\mathcal{D})$ of the network $N$ decreases in this case. For this purpose, define $S_{1}, S_{2}$ and $\Delta_{i}^{\prime}$ $(1 \leqq i \leqq m)$ with respect to $B_{a *}^{0} \varepsilon D^{ \pm}$by

$$
\begin{gathered}
S_{1}=\left\{a \varepsilon A^{\mp}\left(\tilde{T}_{r}^{n}\right) \cap X^{\prime \prime}: \tilde{X}^{n}(a)>b(a)\right\} \cup\left\{a \varepsilon A^{ \pm}\left(\tilde{T}_{r}^{n}\right) \cap X^{\prime \prime}: \tilde{x}^{n}(a)<0\right\}, \\
S_{2}=\left\{a \varepsilon A^{\mp}\left(\tilde{T}_{r}^{n}\right) \cap X^{\prime \prime}: \tilde{x}^{n}(a)<0\right\} \cup\left\{a \varepsilon A^{ \pm}\left(\tilde{T}_{r}^{n}\right) \cap X^{\prime \prime}: \tilde{x}^{n}(a)>b(a)\right\}, \\
\Delta_{i}^{\prime}= \begin{cases}-x^{0}\left(a_{i}^{\prime}\right) & \left(a_{i}^{\prime} \varepsilon A^{\mp}\left(\tilde{T}_{r}^{n}\right)\right), \\
x^{0}\left(a_{i}^{\prime}\right)-b\left(a_{i}^{\prime}\right) & \left(a_{i}^{\prime} \varepsilon A^{ \pm}\left(\tilde{T}_{r}^{n}\right)\right),\end{cases}
\end{gathered}
$$

where $X^{\prime \prime}=A\left(Q^{\prime}\right) \cap A\left(\tilde{P}_{y r}^{n}\right)$ for the path $\tilde{P}_{y r}^{n}$ of $\tilde{T}_{r}^{n}$ and the values $x^{0}\left(a_{i}^{\prime}\right)$ on the arc $a_{i}^{\prime}$ connecting $B_{i}^{\prime}$ to $B_{i+1}^{\prime}, \tilde{x}^{n}(a)$ appearing above are defined in (4.9), (4.10), respectively. Assume that $e^{\prime \prime} \varepsilon S_{1} \cup S_{2}$ is the arc nearest to vertex $y$ and that 
(4.21) $\quad\left(\tilde{x}^{n+1}(a): a \varepsilon A\left(\tilde{T}_{r}^{n+1}\right)\right), \tilde{z}_{r}^{n+1}$ are the values given after doing $\operatorname{ADJUST}\left(\tilde{T}_{r}^{n}, e^{\prime \prime},\left(\tilde{X}^{n}(a): a \varepsilon A\left(\tilde{T}_{r}^{n}\right)\right), \tilde{z}_{r}^{n}\right)$,

where $\tilde{T}_{r}^{n+1}=\tilde{T}_{r}^{n}-V\left(\tilde{B}_{e}^{n} n\right)$ for leaf branch $\tilde{B}_{e}^{n}$ of $\tilde{T}_{r}^{n}$ and $\left(\tilde{x}^{n}(a): a \varepsilon A\left(\tilde{T}_{r}^{n}\right)\right)$, $\tilde{z}_{r}^{n}$ are the values defined in (4.10). Then we have:

Proposition 4.14. If $B_{a *}^{0} \varepsilon D^{ \pm}$and $Q^{\prime} \varepsilon D^{\mp}$, then we have either (1) or (2).

(1) (1-1) If there is some $j$ such that $e^{\prime \prime}=a_{j}^{\prime} \varepsilon S_{1}$, then we have

$$
\begin{array}{ll}
\tilde{x}^{n+1}(a)>0 & \left(a \varepsilon A^{\mp}\left(\tilde{T}_{r}^{n+1}\right) \cap X_{1}\right), \\
\tilde{x}^{n+1}(a)<b(a) & \left(a \varepsilon A^{ \pm}\left(\tilde{T}_{r}^{n+1}\right) \cap X_{1}\right),
\end{array}
$$

(1-2) If there is some $j$ such that $e^{\prime \prime}=a_{j}^{\prime} \varepsilon S_{2}$, then we have

$$
d(\mathscr{D})>d(\tilde{D}) \text { for } \mathscr{D}=\mathscr{D}^{+} \cup \mathscr{D}^{-} \text {and } \tilde{D}^{-}=\tilde{D}^{+} \cup \tilde{D}^{-} \text {, }
$$

(2) If there is some $j$ such that $e^{\prime \prime} \varepsilon A\left(B_{j}^{\prime}\right)$, then we have

$$
\begin{array}{ll}
\tilde{x}^{n+1}(a)>0 & \left(a \varepsilon A^{\mp}\left(\tilde{T}_{r}^{n+1}\right) \cap X_{2}\right), \\
\tilde{x}^{n+1}(a)<b(a) & \left(a \varepsilon A^{ \pm}\left(\tilde{T}_{r}^{n+1}\right) \cap X_{2}\right),
\end{array}
$$

where $X_{1}=A\left(B_{j+1}^{\prime}\right) \cap A\left(\tilde{P}_{y r}^{n}\right)$ and $X_{2}=A\left(B_{j}^{\prime}-V\left(\tilde{B}_{e}^{n} \tilde{u}^{\prime \prime}\right)\right) \cap A\left(\tilde{P}_{y r}^{n}\right)$ for the path $\tilde{P}_{y r}^{n}$ of $\tilde{T}_{r}^{n}$ and the leaf branch $\tilde{B}_{e}^{n}$ " of $\tilde{T}_{r}^{n}$.

Proof: We only prove the case (1) where $A\left(T_{r}^{0}\right)=A^{-}\left(T_{r}^{0}\right)$ and $B_{a^{*} \varepsilon}^{0} D^{+}$. Let $\left(x^{0}(a): a \varepsilon A\left(T_{\Gamma}^{0}\right)\right)$ be the values defined in $(4.9)$.

$(1-1):$ If $e^{\prime \prime}=a_{j}^{\prime} \varepsilon S_{1}$, then we have $\tilde{B}_{e}^{n} n \varepsilon \underline{\mathscr{D}}^{+}$. Let $\Delta_{e} "=b\left(a_{j}^{\prime}\right)-x^{0}\left(a_{j}^{\prime}\right)$, then from Proposition 4.6 and (4.20), it follows that

$$
\tilde{x}^{n+1}(a)=x^{0}(a)+\Delta_{e}{ }^{\prime \prime}>x^{0}(a)+\Delta_{j}^{\prime} \geq 0 \quad\left(a \varepsilon A\left(B_{j+1}^{\prime}\right) \cap A\left(\tilde{P}_{y r}^{n}\right)\right) .
$$

$(1-2)$ : Since $e^{\prime \prime}=a_{j}^{\prime} \varepsilon S_{2}$, we have $G^{\prime} \varepsilon \tilde{D}^{-}$and $d\left(G^{\prime}\right)=\Delta_{j}^{\prime}-\Delta_{a} n$ for some subgraph $G^{\prime}$ of $\tilde{B}_{e}^{n}$. Hence the deficiency change is given as follows.

$$
d(\not)-d(\tilde{D})=\Delta_{k}+\Delta_{j}^{\prime}-\left(\left(\Delta_{k}-\Delta_{a} n\right)+\left(\Delta_{j}^{\prime}-\Delta_{a} n\right)\right)>0 . \square
$$

Proposition 4.14 means what follows. Case (1) (i.e., the arc $e^{\prime \prime}$ equals some $\operatorname{arc} a_{j}^{\prime}$ joining $B_{j}^{\prime}$ and $B_{j+1}^{\prime}$ ) says that only if the leaf branch $\tilde{B}_{e}^{n}$ is in $\underline{D}^{\mp}$ for the leaf branch $B_{a * \varepsilon D^{ \pm}}^{0}$ of ( the old ) $T_{r}^{0}$, then the deficiency reduces. Conversely, for Case (2) (i.e., the arc $e^{\prime \prime}$ is on some $B_{j}^{\prime}$ ), we always have $\tilde{B}_{e}^{n} \varepsilon \underline{\mathscr{D}}^{ \pm}$for $B_{a *}^{0} \varepsilon \mathscr{D}^{ \pm}$. The following proposition shows that the deficiency decreases for two special cases.

Proposition 4.15. If the leaf branch $B_{a^{*}}^{0}$ is in $D^{ \pm}$and $Q^{\prime} \varepsilon D^{\mp}$, then the deficiency decreases in the following two cases (1) and (2). Case (1): $Q^{\prime}$ equals ( the new ) $T_{r}^{0}$.

Case (2): $Q^{\prime}$ equals some leaf branch $B_{a}^{0}$, of ( the new ) $T_{r}^{0}$ and that we have at least one member of $\underline{\tilde{D}}^{\mp}$ from $Q^{\prime}$.

Proof: We only prove Case (1) where $B_{a * \varepsilon}^{0} \in D^{+}, A\left(T_{r}^{0}\right)=A^{-}\left(T_{r}^{0}\right)$ and $S_{1} \cup S_{2}$ (defined in $(4 \circ 18),(4.19)$ ) is empty. Let $z_{r}^{0}, \tilde{z}_{r}^{n}$ be the values defined in 
(4.9), (4.10), respectively. If $z_{r}^{0}>\Delta_{a} n$ for $\Delta_{a} n$ defined in (4.8), then we have $\tilde{T}_{r}^{4} \varepsilon \underline{\mathbb{D}}-d\left(\tilde{B}_{a}^{0}\right)=\Delta_{k}-\Delta_{a} n$ and $d\left(\tilde{T}_{r}^{4}\right)=z_{r}^{0}-\Delta_{a} n$ where $\tilde{B}_{a}^{0}$ is the leaf branch of $\tilde{T}_{r}^{0}$ and $\tilde{T}_{r}^{4} \supset Q^{\prime}$ is the rooted subtree of $\tilde{T}_{r}^{n}$. Therefore, the deficiency change is computed as

$$
d(\mathscr{D})-d(\tilde{D})=\Delta_{k}+z_{r}^{0}-\left(\left(\Delta_{k}-\Delta_{a} n\right)+\left(z_{r}^{0}-\Delta_{a} n\right)\right)>0 .
$$

Note here that we have $\Delta_{a} n>0$. If $z_{r}^{0}=\Delta_{a} n$, then put $z_{r}^{0}=\Delta_{a} n$ in (4.24). On the other hand, if $z_{r}^{0}<\Delta_{a} n$, then from $\tilde{z}_{r}^{n}=z_{r}^{0}-\Delta_{a}^{n}$ and $\tilde{T}_{r}^{5} \varepsilon \underline{D}^{+}$for some rooted subtree $\tilde{T}_{r}^{5}$ of $\tilde{T}_{r}^{n}$, it follows that

$$
d(\varnothing)-d(\tilde{D})=\Delta_{k}+z_{r}^{0}-\left(\left(\Delta_{k}-\Delta_{a} n\right)+\left(\Delta_{a} n-z_{r}^{0}\right)\right)>0 \text {. }
$$

Note here that we have $z_{r}^{0}>0$. $\square$

Now, the only case remained to be checked is the one where for the leaf branch $B_{a}^{0} \varepsilon D^{ \pm}$of ( the old ) $T_{r}^{0}, Q^{\prime} \varepsilon D^{\mp}$ equals the leaf branch $B_{a}^{0}$, of ( the new ) $T_{r}^{0}$ and that we have no member $M \subset Q^{\prime}$ of $\underline{D}^{\mp}$. In this case we can similarly prove $d(D)>d(\tilde{D})$. Hence, we have the following proposition.

Proposition 4.16. We have $d(D)>d(\tilde{D})$ for $B_{a^{*} \varepsilon}^{0} \varepsilon D^{ \pm}$and $Q^{\prime} \varepsilon D^{\mp} . \square$

We have proved the previous propositions $4.8 \sim 4.16$ by assuming that the set $U^{ \pm}$defined in (4.4) is not empty, but it is also easy to see that we have the propositions 4.10 4.16 for empty set $U^{ \pm}$.

Let $p=|D|, q=\Sigma\{|V(Q)|: Q \varepsilon D\}, \tilde{p}=|\tilde{D}|$ and $\tilde{q}=\Sigma\{|V(\tilde{Q})|: \tilde{Q} \varepsilon \tilde{D}\}$. ( $p$ is the number of members of $D$, while $q$ is the number of vertices contained in those of $D$. ) From Propositions 4.10 4.13, it follows that if the leaf branch $B_{a}^{0 * \varepsilon} D^{ \pm}$and $Q^{\prime} \varepsilon D^{ \pm} \cup \mathcal{N}$, then the deficiency $d(\mathcal{D})$ does not change but that $p$ and $q$ are monotone functions of the number of pivot operations where the value $p+|V|-q$ is reduced at least one by a pivot operation. Hence we have:

Proposition 4.17. For $B_{a}^{0} \varepsilon \varepsilon D^{ \pm}$and $Q^{\prime} \varepsilon D^{ \pm} \cup \mathcal{N}$, we have $d(D)=d(\tilde{D}), \quad p \geqq \tilde{p},|V|-q \geqq|V|-\tilde{q}$, where $(p,|V|-q) \neq(\tilde{p},|V|-\tilde{q}) \cdot \square$

We are now ready to estimate the computational complexity of the algorithm.

Theorem 4.18. The algorithm requires at most $(2|V|-4)(|V|-1) d(\varnothing)$ pivot operations and its running time is $O\left(|V|^{2}|A| d(D)\right)$.

Proof: The deficiency function $d$ is nonnegative by definition. Since $b(a)$ and $x(a)(a \varepsilon A)$ are integral, $d(D)$ is also integral. We have seen that $d$ is non-increasing and, if $d(D)=d(\tilde{D})$, then $p+|V|-q$ is reduced at least 1 from Proposition 4.17. Consider the number of pivot operations except Trimming operations, to decrease the value $d(D)$. Now let $p^{(k)}$ (resp. $q^{(k)}$ ) be the number of the members of $D$ (resp. that of vertices contained in 
those of $D$ ) after $k$ pivot operations. Assume that we initially have $p^{(0)}=p$ and $q^{(0)}=q$. Then from $p \leqq q \leqq|V|$, we have $(p-1)+(|V|-q) \leqq|V|-1$. This means that at most $|V|-1$ pivot operations are required except Trimming operations. From Proposition 4.3 we have at most $(2|V|-4)(|V|-1)$ pivot operations to reduce $d(D)$. If $d(D)=0$, then the algorithm is over. So, the total number of pivot operations is $(2|V|-4)(|V|-1) d(D)$. As to the running time, we require $O(|A|)$ time in Steps 0 and $3, O(|V|)$ time in Steps 1,2 and 4 and $O(|A||V|)$ time in Step 5. Hence the total running time is $O\left(|V|^{2}|A| d(D)\right)$. $\square$

\section{Application of Scaling Method}

We express upper capacities $b(a)$ (acA) in binary form. Then we consider a sequence of problems each of which has approximated capacities. Scaling method is the new way to get an optimal solution for the original problem $(P)$ by solving a sequence of these approximated problems repeatedly. Let (5.1) $\quad t=\max \left\{i+1: 2^{i} \leqq \max \{b(a): a \varepsilon A\}<2^{i+1}\right\}$.

Define, for each $a \varepsilon A, b^{(k)}(a)=\Gamma_{b}(a) / 2^{t-\bar{k}} \quad(0 \leqq k \leqq t)$, where for a number $n$, $\lceil\eta\urcorner$ is the minimum integer greater than or equal to $n$. Now, we consider the following minimum cost flow problem $\mathrm{P}(k)$ :

$$
\begin{array}{rlrl}
\mathrm{P}(k): \min & \{\{c(a) x(a): a \varepsilon A\}, & \\
& \sum\left\{x(a): a \varepsilon \delta^{+} v\right\}-\sum\left\{x(a): a \varepsilon \delta^{-} v\right\}=0 \quad & & (v \varepsilon V), \\
& \leq x(a) \leqq b(k)(a) & & (a \varepsilon A) .
\end{array}
$$

We also define the deficiency functions $\tilde{d}_{k}$ and $d_{k}$ with respect to Problem $\mathrm{P}(k)$ similarly as $\tilde{d}$ and $d$. Let $\bar{x}^{(k)}$ be an optimal solution of the problem $P(k)$. Our aim here is to estimate the deficiency $d_{k}(D)$. Since $b^{(0)}(a)=1$ for each $a \varepsilon A, d_{0}(D)$ is bounded as follows.

Proposition 5.1. For Problem $P(0)$, we have $d_{0}(\hat{D}) \leqq 2(|V|-1)|A|$.

Proof: From $b^{(0)}(a)=1(a \varepsilon A)$ and Tree partitioning algorithm of $T_{r}$, we have $d_{0}(Q) \leqq|A|$ for each $Q \varepsilon \mathcal{D}$ such that $Q$ is either a leaf branch or $\mathcal{N}$ surrounded graph of $T_{r}$. Assume that $Q$ is in $D^{+}$and $Q^{\prime} \varepsilon D^{-}$is the nearest to Q. Denote $X\left(Q^{\prime}\right)$ by

(5.2) $X\left(Q^{\prime}\right)=\left\{\tilde{Q} \varepsilon D^{+}: \tilde{Q}\right.$ is contained in some component of $T_{r}-V\left(Q^{\prime}\right)$ without root $r\}$.

Then we have $d_{0}\left(Q^{\prime}\right) \leqq \Sigma\left\{d_{0}(\tilde{Q}): \tilde{Q} \varepsilon X\left(Q^{\prime}\right)\right\}+|A|$. Note that the members of $D^{+}$and those of $D^{-}$appear from root $r$ to each leaf of $T_{r}$, alternatively, if we ignore members of $\mathcal{N}$. Let $x$ be the circulation in Section 2.1 obtained from $T_{r}$ for the problem $P(0)$. Then $d_{0}(D)$ is not more than the sum of two times the difference between the value $x\left(a^{\prime \prime}\right)$ and its upper or lower bound, where $a^{\prime \prime}$ is the tree arc joining directly or indirectly between the member of $\mathcal{D}^{+}$ 
and that of $D^{-}$. (i.e., a" may be joining a member of $D$ and that of $\mathcal{N}_{\text {.) }}$ The number of such $a^{\prime \prime}$ is at most $|V|-1$.

Next, we make some preparations for finding an upper bound of $d_{k}(D)$ $(k \geqq 1)$. We have the following relation:

$$
2 b^{(k-1)}(a)-1 \leqq b(k)(a) \leqq 2 b(k-1)(a)
$$

$(a \varepsilon A)$.

Let $T_{r}^{(k-1)}$ be a rooted spanning tree from which an optimal solution for Problem $\mathrm{P}(k-1)$ is obtained. Such a tree is called an optimal tree. Let $p^{(k-1)}$ be the potential determined by $T_{r}^{(k-1)}$. Then for Problem $\mathrm{P}(k)$, the potential $p^{(k-1)}$ satisfies the condition $(C S)^{*}$. Now, we also define Problem $\tilde{\mathrm{P}}(k)$ similar to Problem $\mathrm{P}(k)$, i.e., $b^{(k)}$ given in the problem $\mathrm{P}(k)$ is replaced by $2 b(k)$ for the problem $\tilde{\mathrm{P}}(k)$. Once we find an optimal solution $\bar{x}^{(k-1)}$, an optimal one $\tilde{x}^{(k-1)}$ for the problem $\tilde{\mathrm{P}}(k-1)$ is easily obtained because we may choose $T_{r}^{(k-1)}$ as an optimal tree. For the problem $\mathrm{P}(k)$, we pick up $T_{r}^{(k-1)}$ as an initial tree. Let ${ }_{x}(k)$ be the circulation determined from $T_{r}^{(k-1)}$ in Section 2.1. For an arc $a(a \varepsilon A)$ and the leaf branch $B_{a}^{(k-1)}$ of $T_{r}^{(k-1)}$, define $A_{i}(1 \leqq i \leqq 4)$ by

(5.4) $A_{1}=\left\{e \varepsilon A-A\left(T_{r}^{(k-1)}\right): \tau\left(p^{(k-1)}, e\right) \geqq 0, \partial^{+} e \varepsilon V\left(B_{a}^{(k-1)}\right), \partial^{-} e \varepsilon V-V\left(B_{a}^{(k-1)}\right)\right\}$,

(5.5) $A_{2}=\left\{e \varepsilon A-A\left(T_{r}^{(k-1)}\right): \tau\left(p^{(k-1)}, e\right)<0, \partial^{+} e \varepsilon V\left(B_{a}^{(k-1)}\right), \partial^{-} e \varepsilon V-V\left(B_{a}^{(k-1)}\right)\right\}$,

(5.6) $A_{3}=\left\{e \varepsilon A-A\left(T_{r}^{(k-1)}\right): \tau\left(p^{(k-1)}, e\right) \geqq 0, \partial^{-} e \varepsilon V\left(B_{a}^{(k-1)}\right), \partial^{+} e \varepsilon V-V\left(B_{a}^{(k-1)}\right)\right\}$,

(5.7) $A_{4}=\left\{e \varepsilon A-A\left(T_{r}^{(k-1)}\right): \tau\left(p^{(k-1)}, e\right)<0, \partial^{-} e \varepsilon V\left(B_{a}^{(k-1)}\right), \partial^{+} e \varepsilon V-V\left(B_{a}^{(k-1)}\right)\right\}$.

$A_{i}(1 \leqq i \leqq 4)$ is illustrated in Fig. 11 .

$T_{r}^{(k-1)}$ :

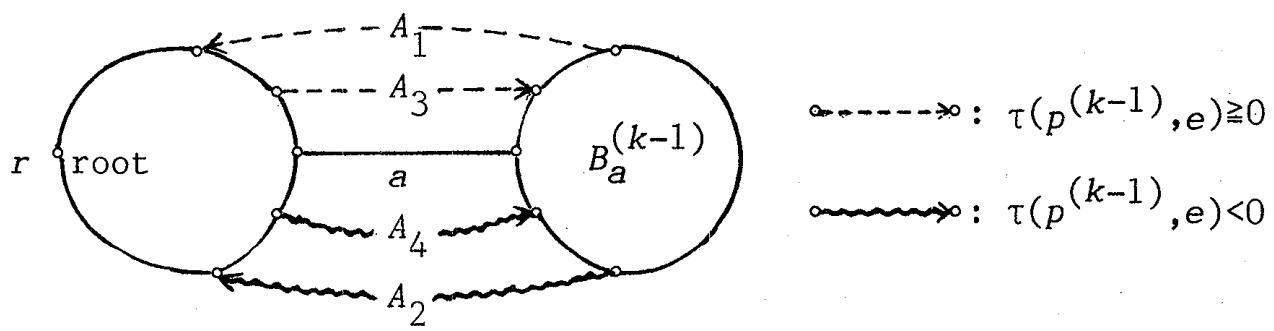

Fig.11

Then the following proposition estimates the difference between the value ${ }_{x}^{(k)}(a)$ and its upper or lower bound for $a \varepsilon A\left(T_{r}^{(k-1)}\right)$.

Proposition 5.2. For each $a \varepsilon A\left(T_{r}^{(k-1)}\right)(k \geqq 1)$, we have $\max \left\{-x^{(k)}(a), x^{(k)}(a)-b^{(k)}(a)\right\} \leq \max \left\{\left|A_{1}\right|+\left|A_{2}\right|,\left|A_{3}\right|+\left|A_{4}\right|\right\}+1$. 
Proof: Assume that $A\left(T_{r}^{(k-1)}\right)=A^{+}\left(T_{r}^{(k-1)}\right)$. It suffices to show that for the arc $a \varepsilon A\left(T_{r}^{(k-1)}\right)$ not satisfying the capacity condition, we have

$$
\begin{aligned}
& x^{(k)}(a)-b^{(k)}(a) \leqq\left|A_{3}\right|+\left|A_{4}\right|+1, \\
& { }_{-x}{ }^{(k)}(a) \leqq\left|A_{1}\right|+\left|A_{2}\right|+1 .
\end{aligned}
$$

We check (1) only. Let $A_{i}^{\prime}=\left\{e \varepsilon A_{i}: \tilde{x}^{(k-1)}(e)=2 b(k-1)(e)\right\} \quad(i=1,3)$. Then from an optimal solution $\tilde{x}^{(k-1)}$, we have

$$
\begin{aligned}
& \sum\left\{2 b^{(k-1)}(e): e \varepsilon A_{2}\right\}+\sum\left\{\tilde{x}^{(k-1)}(e): e \varepsilon A_{1}^{\prime}\right\} \\
& =\tilde{x}^{(k-1)}(a)+\sum\left\{2 b^{(k-1)}(e): e \varepsilon A_{4}\right\}+\sum\left\{\tilde{x}^{(k-1)}(e): e \varepsilon A_{3}^{\dagger}\right\},
\end{aligned}
$$

(5.9) $\quad 0 \leqq \tilde{x}(k-1)(a) \leqq 2 b(k-1)(a)$.

As $x^{(k)}$ is a circulation in the problem $\mathrm{P}(k)$, we have

(5.10) $\quad \sum\left\{b^{(k)}(e): e \varepsilon A_{2}\right\}+\Sigma\left\{b^{(k)}(e): e \varepsilon A_{1}^{\prime}\right\}$

$$
=x^{(k)}(a)+\sum\left\{b^{(k)}(e): e \varepsilon A_{4}\right\}+\sum\left\{b^{(k)}(e): e \varepsilon A_{3}^{1}\right\} \text {. }
$$

From (5.3) and (5.8) (5.10), it follows that

$$
\begin{aligned}
& x^{(k)}(a)-b^{(k)}(a) \leqq \Sigma\left\{2 b^{(k-1)}(e): e \varepsilon A_{2}\right\}-\sum\left\{\left(2 b^{(k-1)}(e)-1\right): e \varepsilon A_{4}\right\} \\
& +\sum\left\{2 b^{(k-1)}(e): e \varepsilon A_{1}^{\prime}\right\}-\sum\left\{\left(2 b^{(k-1)}(e)-1\right): e \varepsilon A_{3}^{\prime}\right\} \\
& -\left(2 b^{(k-1)}(a)-1\right) \\
& \leqq \tilde{x}^{(k-1)}(a)+\left|A_{4}\right|+1-2 b^{(k-1)}(a)+\left|A_{3}\right| \\
& \leqq\left|A_{3}\right|+\left|A_{4}\right|+1 \text {. }
\end{aligned}
$$

The following proposition, which gives an upper bound of $d_{k}(\mathscr{D})$, can be shown by using Propositions 5.1 and 5.2.

Proposition 5.3. For Problem $\mathrm{P}(k)(k \geqq 1)$, the deficiency is bounded as $d_{k}(D) \leqq 2(|V|-1)|A|$.

From Theorem 4.18, Propositions 5.1 and 5.3, a polynomial-time algorithm for Problem $(P)$ is given by solving a sequence of $(t+1)$ subproblems $\mathrm{P}(k)(0 \leqq k \leqq t)$. Hence we have the following theorem.

Theorem 5.4. We can obtain an optimal solution for the minimum cost flow problem (P) by the repetition of at most $4(|V|-2)(|V|-1)^{2}|A|(t+1)$ pivot operations. $\square$

\section{References}

[1] Balinski, M. L. and Gomory, R. E.: A Primal Method for the Assignment and Transportation Problems. Management Science, Vo1.10, No.3 (1964), 578-593.

[2] Cunningham, W. H.: A Network Simplex Method. Mathematical Programming, Vo1.11 (1976), 105-116. 
[3] Dantzig, G. B.: Linear Programming and Extensions. Princeton University Press. Princeton, N.J., 1963.

[4] Edmonds, J. and Karp, R. : Theoretical Improvements in Algorithmic Efficiency for Network Flow Problems. Journal of the ACM, Vol.19 (1972), 248-264.

[5] Ford, L. R. Jr. and Fulkerson, D. R.: A Primal Dual Algorithm for the Capacitated Hitchcock Problem. Naval Research Logistics Quarterly, Vo1.4 (1957), 47-54.

[6] Ford, L. R. Jr. and Fulkerson, D. R.: Flows in Networks. Princeton University Press. Princeton, N.J., 1962.

[7] Fujishige, S.: A Capacity-rounding Algorithm for the Minimum-cost Circulation Problem ---- A Dual Framework of the Tardos Algorithm. Mathematical Programming, Vo1.35, No.3 (1986), 298-308.

[8] Ikura, Y. and Nemhauser, G. L.: A Polynomial-time Dual Simplex Algorithm for the Transportation Problem. School of Operations Research and Industrial Engineering, Cornel1 University, 1984.

[9] Ikura, Y. and Nemhauser, G. L.: Computational Experience with a Polynomial-time Dual Simplex Algorithm for the Transportation Problem. Discrete Applied Mathematics, Vol.13 (1986), 239-248.

[10] Iri, M.: A New Method of Solving Transportation Network Problems。 Journal of the Operations Research Society of Japan, Vol.3 (1960), 27-87.

[11] Klein, M.: A Primal Method for Minimal Cost Flows with Applications to the Assignment and Transportation Problems. Management Science, Vo1.14 (1967), 205-220.

[12] Lawler, E. L.: Combinatorial Optimization - Networks and Matroids. Ho1t, Rinehart and Winston, New York, 1976.

[13] Orlin, J. B.: Genuinely Polynomial Simplex and Non-simplex Algorithms for the Minimum Cost Flow Problem. Working Paper No.1615-84, Alfred P. Sloan School of Management, MIT, December, 1984.

[14] Takahashi, I. and Fujishige, S.: Discrete Mathematics (in Japanese). Information Science, Vol.17, Iwanami Shoten, 1981.

[15] Tardos, E.: A Strong1y Polynomial Minimum Cost Circulation Algorithm. Combinatorica, Vol.5, No.3 (1985), 247-255.

Akira NAKAYAMA: Department of Management Sciences, Faculty of Commerce, Otaru University of Commerce, Midori, Otaru, Hokkaido, 047, Japan. 


\title{
最小費用流問題に対する多項式時間の双対単体法
}

\author{
小樽商科大学 中 山 明
}

1984年に，伊倉とNemhauserはヒッチコック型の輸送問題に対して双対単体法による多項式時間ア ルゴリズムを提案している。

一般的な最小費用流問題がヒッチコック型の輸送問題に帰着されるという事実により，伊倉一 Nemhauser のアルゴリズムは，また同時に，最小費用流問題を解くことができる。

本論文では，最小費用流問題を解くにあたって，その問題をヒッチコック型の翰送問題に帰着させ るのではなく，次の立場からアルゴリズムを求める。つまり，伊倉一 Nemhauser のアイデアを直接， 一般のネットワークに拡張し，最小費用流問題を解くための双対単体法による多項式時間アルゴリズ ムを提案する。 